\title{
(2) \\ Dormancy and Revitalization: The fate of ethnobotanical knowledge of camel forage among Sahrawi nomads and refugees of Western Sahara
}

\author{
Gabriele Volpato and Rajindra K. Puri
}

\section{Research}

\begin{abstract}
Knowledge about forage is fundamental to the survival of pastoral populations around the world. In this paper, we address the knowledge of camel forage of Sahrawi nomads and refugees of Western Sahara. We analyze the distribution of this knowledge through cultural consensus analysis and develop an explanation for intra-cultural variation based on changing processes of knowledge transmission. In total, 100 plant species were free-listed by informants, with five species (i.e., Acacia tortilis (Forssk.) Hayne, Nucularia perrinii Batt., Astragalus vogelii (Webb) Bornm., Panicum turgidum Forssk., and Stipagrostis plumosa Munro ex T.Anderson) found to be culturally highly salient. These five represent five local categories of forage that are necessary for camel management in the Western Sahara desert. The Sahrawi listed 25 forage plants that influence the taste and properties of camel milk, demonstrating that cultural values, as much as survival functions, underpin local knowledge systems. Perhaps unsurprisingly, age and nomadic experience are positively correlated with forage knowledge. Forced displacement and sedentarization are hypothesized as causes of progressive non-use of this knowledge and the lack of its transmission to younger generations of refugees. Nonetheless, across the study area, refugees are re-engaging with pastoralism and nomadism, which is leading to a revitalization of forage knowledge and its transmission. This should be regarded as an adaptation pathway for refugees.
\end{abstract}

\section{Resumen}

Para las poblaciones pastorales de todo el mundo, el conocimiento de las plantas forrajeras es fundamental para su supervivencia. En este trabajo se aborda el conocimiento sobre forraje de camellos entre los nómadas y los refugiados saharauis del Sáhara Oc- cidental. Se analiza también la distribución de estos conocimientos a través de un análisis de consenso cultural, y se desarrolla una explicación para la variación intra-cultural basada en el cambio de los procesos de transmisión de conocimientos entre los refugiados. En total, 100 especies de plantas fueron enumeradas por los informantes, con cinco especies (Acacia tortilis (Forssk.) Hayne, Nucularia perrinii Batt., Astragalus vogelii (Webb) Bornm., Panicum turgidum Forssk., and Stipagrostis plumosa Munro ex T.Anderson) culturalmente muy relevantes. Las plantas forrajeras se clasifican en cinco categorías que son necesarias para la gestión de camellos en el desierto del Sahara occidental. Los saharauis reconocen 25 plantas forrajeras que influyen en el sabor y las propiedades de la leche de camella, hecho que muestra cómo los valores culturales, tanto como las funciones de supervivencia, sustentan los sistemas de conocimientos locales. Tal como era de esperar, la edad y la experiencia nómada están correlacionadas con el conocimiento de plantas forrajeras. El desplazamiento y

\section{Correspondence \\ Gabriele Volpato, Department of Social Sciences, Wagenin- gen University, THE NETHERLANDS. gabriele.volpato@wur.nl \\ Rajindra K. Puri, Centre for Biocultural Diversity, School of Anthropology and Conservation, University of Kent at Canterbury, Canterbury, UNITED KINGDOM. \\ Ethnobotany Research \& Applications 12:183-210 (2014)}

Published: 26 June 2014 
la sedentarización forzada parecen ser la causa de la progresiva falta de utilización y transmisión de estos conocimientos a las generaciones más jóvenes de los refugiados. Sin embargo, dentro de un proceso más amplio de re-pastoralización de los refugiados, estos conocimientos están siendo reactivados y otra vez transmitidos a través de un proceso de revitalización que debe considerarse como una vía de adaptación de los refugiados.

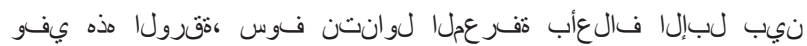

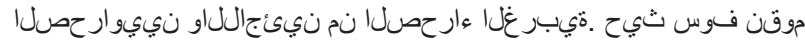

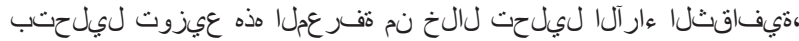

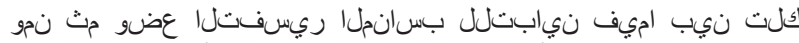

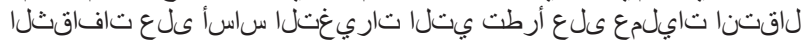

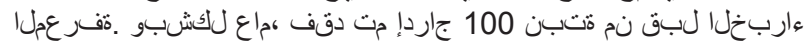

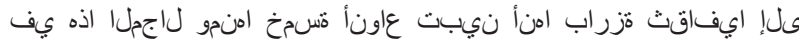
يمو ،ريبك دح : Acacia tortilis (Forssk.) Hayne، Nucularia perrinii Batt.، Astragalus vogelii (Webb) Bornm.، Panicum turgidum Forssk. ' Stipagrostis plumosa Munro ex

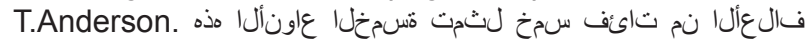

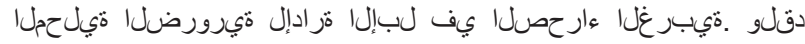

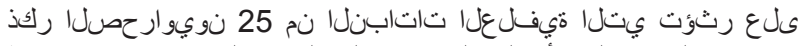

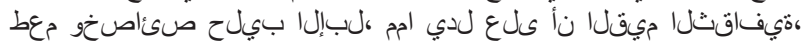

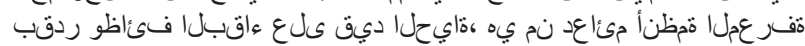

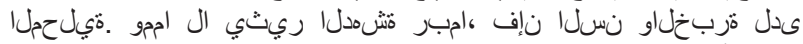

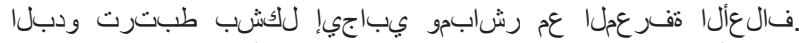

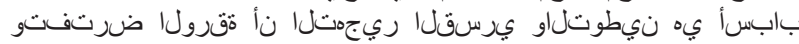

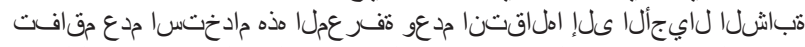

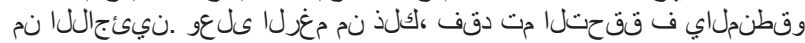

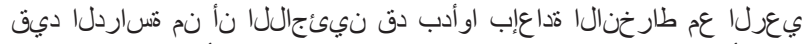

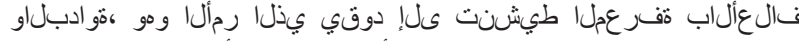

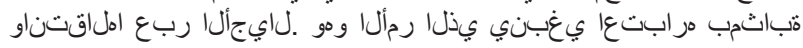

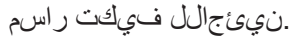

\section{Introduction}

Ethnobotanical knowledge is crucial to the production and survival of human populations around the world (Anderson et al. 2011). It is particularly central to food security among populations that depend directly on the production and harvesting of food for survival (e.g., small farmers, nomads, pastoralists, and hunter-gatherers), to traditional food-producing systems (Scoones et al. 1992), and to people who strive to diversify their livelihoods to meet their material and cultural needs (e.g., migrants and refugees; see Pieroni \& Vandebroek 2007). Indeed, ethnobotanical knowledge is often rich, detailed, and adaptive (Berkes et al. 2000), and it deserves as such to be recorded and its cultural transmission promoted (Cavalli-Sforza et al. 1982, Ellen et al. 2013). Although the literature often stresses that loss of ethnobotanical knowledge occurs within wider processes of globalization, urbanization, and displacement (Folke 2004, Turner \& Turner 2008, Zent 2001), few studies have addressed the processes that occur with inactivity (knowledge dormancy) and revitalization of such knowledge for survival or cultural reasons and the resumption of transmission among certain groups (Ohmagari \& Berkes 1997).

Within the corpus of plant knowledge held by pastoral populations across the world, ethnobotanical knowledge about forage and its characteristics is among the most fundamental to survival (Fernandez-Gimenez 2000). In this paper, we use the term "forage" to indicate plants grazed or browsed by camels directly, whereas "pasture" indicates the land with vegetation cover used by camels within the Sahrawi pastoral system. Livestock husbandry is possible only through the rational exploitation of grassland, mountain, or desert pastoral resources, and this cannot occur without detailed knowledge of these resources. The importance of this knowledge becomes even more crucial among camel pastoralists living in desert environments (Johnson 1993). In fact, "the science of pastures is a difficult art" (Caratini 1989b:36) as it requires in-depth knowledge of camel physiology and ethology, of the local vegetation (i.e., species diversity, abundance, distribution, growth rates, forage qualities, and camel preferences), and of climatic patterns, places, paths, and distances.

In this paper, we describe Sahrawi knowledge of camel forage in Western Sahara and discuss the role of this knowledge in the production and reproduction of Sahrawi society. We argue that the Sahrawi have a detailed knowledge of camel forage and its properties within their nomadic territories, that this knowledge is pivotal to their mode of subsistence based on camel husbandry and nomadism, and that it is underpinned by cultural values. With forced sedentarization in refugee camps and widespread loss of camel herds, this knowledge has become largely inactive although not lost in a theoretical sense. We posit that in contexts of changes in culture and productive activities, transmission of ethnobotanical knowledge may be impaired, leading to its dormancy, i.e., it is held by former practitioners but not used and barely transmitted. However, this dormant knowledge may be revitalized and its transmission recovered once it again becomes of material or cultural importance among people who re-engage with past livelihood and environmental management strategies. Such revitalization is occurring among the Sahrawi as refugees re-engage with pastoralism and seasonal nomadism within a wider process of livelihood diversification and cultural revitalization. We finally argue that the inability to transmit this knowledge to younger refugees might prevent their return to pastoralism and nomadism as livelihood strategies, particularly important today as food aid has decreased in recent years (Abdelrahim 2013). Processes of knowledge revitalization can be understood as adaptation pathways for Sahrawi populations (sensu Thornton \& Manasfi 2010).

This paper first presents background information and research methods used in the study, after which follow the results and discussion of Sahrawi knowledge of camel forage that are presented in four parts: (1) the cultural domain of camel forage; (2) folk classification of camel for- 


\section{Volpato \& Puri - Dormancy and Revitalization: The fate of ethnobotanical knowledge of camel forage among Sahrawi nomads and refugees}

age; (3) influence of different forage plants on camel milk and meat taste, smell, and nutritional and medicinal properties; and (4) distribution and transmission of knowledge on camel forage among contemporary Sahrawi. Besides addressing the little-known ethnobotanical knowledge of the Sahrawi people, this study explores the dynamics of knowledge dormancy and revitalization among refugees, which sheds light on the general processes underlying transformations in ethnobotanical knowledge in other populations that have been either displaced in refugee camps or settled in new lands as immigrants.

\section{Background}

Sahrawi, literally "people from the desert," is the autonym of the nomadic and pastoral people who traditionally inhabited coastal areas of Northwestern Africa including Western Sahara, northern Mauritania, and part of south- western Algeria (Figure 1). The Sahrawi people were essentially nomadic, pasturing camels, goats, and sheep in the low-lying plains of Western Sahara and relying for food on livestock products as well as on dates, sugar, cereals, and legumes bartered for livestock in markets peripheral to their nomadic areas (Caratini 1989a,b, Caro Baroja 1955). In 1975, following Morocco's occupation of Western Sahara, about 70,000 Sahrawi fled the Moroccan army (San Martin 2010), becoming refugees. Today, after 16 years of war between Morocco and the Sahrawi's armed political organization-the Polisario Front (19751991) _and the exclusion of refugees from most Western Sahara territory by means of a Moroccan-built wall (known as the berm) that cuts through Western Sahara in a north-south direction, about 165,000 Sahrawi live in four refugee camps (El Aaiún, Smara, Auserd, and Dakhla) located on a desert plateau called Hamada, near the Algerian town of Tindouf (Figure 1).

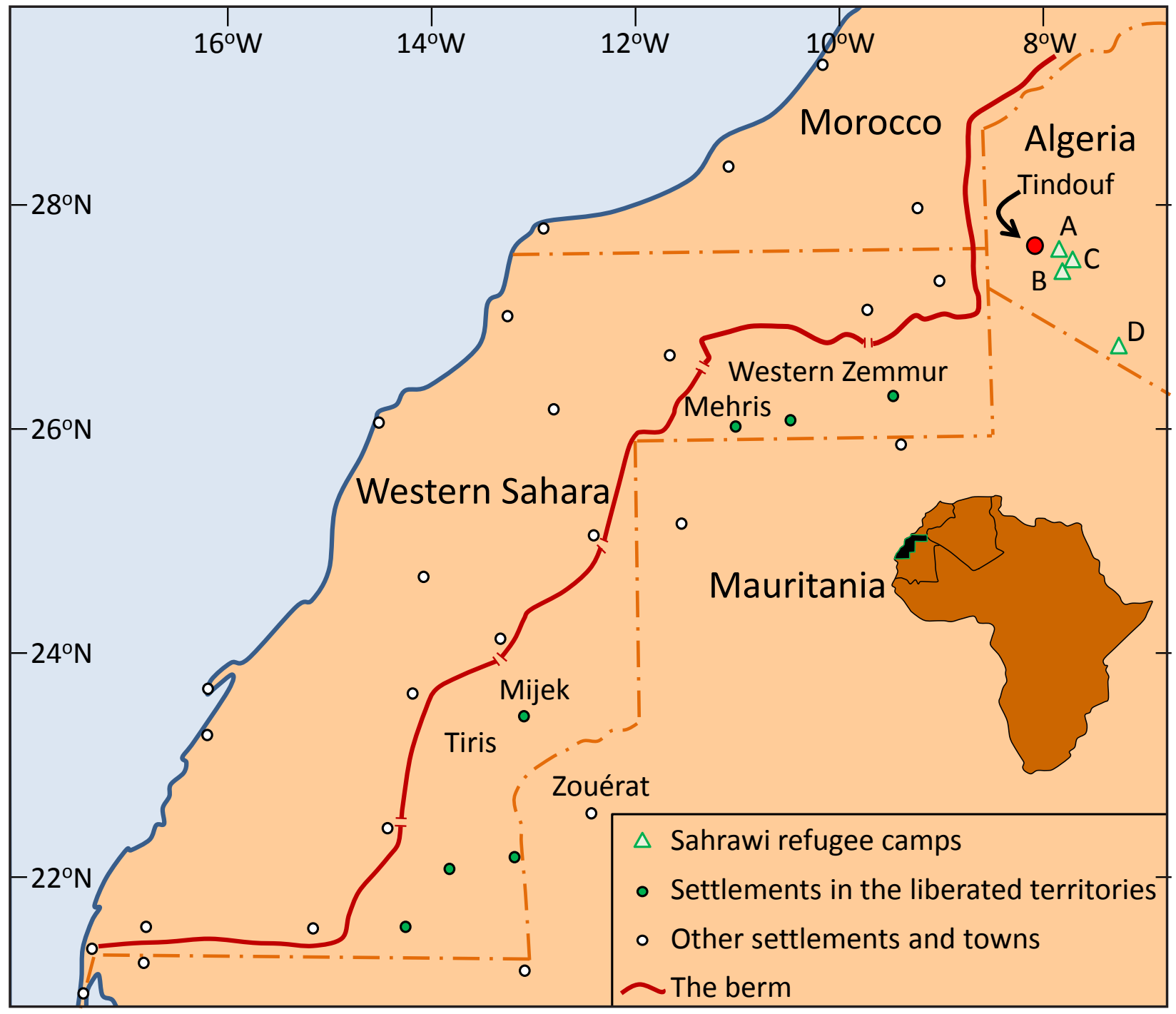

Figure 1. Traditional Sahrawi inhabited coastal areas of Northwestern Africa including Western Sahara, northern Mauritania, and part of southwestern Algeria. Sahrawi refugee camps: A) El Aaiún, B) Smara, C) Auserd, D) Dakhla. 
In the camps, refugees live in tents and mud brick huts and experience problems with water and food supply, using car batteries as the main source of electricity. The European Union, some bilateral development programs, United Nations agencies, Algeria, and several solidarity groups provide food, shelter, and other basic commodities (San Martin 2010). Seeking to improve the quality of life in the camps, refugees have, over the years, developed an informal economy where they market a variety of products (from clothing to personal hygiene products, as well as food), to supplement the diet provided by humanitarian assistance. They engage in some kind of remunerated labor (e.g., as butchers, mechanics, and construction workers) and have expanded trading routes through the camps from Mali, Mauritania, Algeria, and Spain (Bhatia 2001, Dedenis 2005, Herz 2013). Economic activity has increased greatly since the Peace Agreement of 1991 and the subsequent demobilization of Polisario soldiers, who have moved back to the camps and begun to re-engage in livestock husbandry, seasonal nomadism, and trade. Some Sahrawi have migrated abroad and send remittances home.

Besides the camps, through their political representative, the Polisario Front, the Sahrawi have assumed political control over the eastern part of Western Sahara, which was re-gained from Morocco through a guerrilla war that lasted up to the signing of a peace agreement in 1991 (Bhatia 2001). These inland areas of Western Sahara are the so-called "liberated territories" (approximately 20\% of the total area), while the remaining "occupied territories" are under the administrative authority of the Moroccan government. Pastoral areas within the liberated territories are important to the refugees' struggle to maintain or recover traditional livelihoods and cultural and social practices, from livestock husbandry to medicinal plant use (Broglia \& Volpato 2008, Volpato et al. 2012), as well as to earn an income (Volpato et al. 2013). These territories are also inhabited by $20,000-30,000$ Sahrawi nomads who cross them with their herds and use the refugee camps at Tindouf and Zouérat as their main commercial hubs. These nomads survived the war by maintaining their herds and moving to safer areas in Mauritania and Mali; they have progressively returned to the liberated territories after the Peace Agreement of 1991 made it safe to do so.

However, with war, most nomads were driven from their desert lands into refugee camps. Camel herds, having been already weakened and reduced by the drought of the early 1970s, were lost to bombings or abandoned in the desert. There were no livestock in the camps and no farming was possible, so refugees were solely dependent on food aid. With time, knowledge about camel herding slowly declined through lack of use and the death of the elderly population. However, during the 1990s, some refugees managed to obtain an income (e.g., from remittances, Spanish civil service pensions, and commercial activi- ties) and, given the renewed access to part of the former nomadic territories and a decade of good precipitation, began to rebuild herds, primarily of more affordable goats and sheep, but also of camels. Presently, after the autumn rains, thousands of refugee families move with their herds to live in the liberated territories as nomads until the dry season returns (i.e., from September to February). Thus, refugees reduce their dependence on food aid by recovering a lost and much-desired way of life.

This revitalization has been supported by the renewed interactions between refugees and nomads, which have led to a degree of fluidity between these categories. Both groups are using the liberated territories (especially northern areas) to graze herds, and a vast network of solidarity and reciprocity is present in their interactions, which is strengthened by ties of kinship linking nomadic and refugee households. Today, camel husbandry is practiced in the liberated territories as well as in the surroundings of the refugee camps, albeit to a lesser extent. Less than 2000 camels are raised in the camps, whereas some 40,000 head are raised in the liberated territories (DNVRASD 2007). Furthermore, the Polisario own and care for some 27,000 camels, where soldiers or paid laborers act as shepherds (Broglia \& Volpato 2008). In the refugee camps, camel owners supplement natural forage, which is poor on the Hamada plateau, with fodder purchases from Tindouf. In contrast, camel husbandry in the liberated territories that is practiced by both full-time and seasonal nomads, as well as the Polisario, is extensive-that is, highly dependent on pasture areas and mobility for herd production and reproduction. Movements of nomads and their herds are carefully planned and managed, conceptualized as rataa that, in Sahrawi terminology, means "following the pastures."

Across Western Sahara and the liberated territories, the climate is arid and continental, with summer daytime temperatures surpassing $50^{\circ} \mathrm{C}$, while winter temperatures drop to $0^{\circ} \mathrm{C}$ at night. Rainfall can be unpredictable and patchy and generally occurs from the end of the summer through autumn. These rains represent the extreme northerly penetration of the African monsoon from the south or are associated with the Atlantic Westerly from the west (Brooks et al. 2005). Average annual rainfall is 30-50 mm, but rains are highly irregular within and between years, with recurrent droughts. Biogeographically, there are three main areas that fall within this study: (1) the Tindouf Hamada; (2) western Zemmur; and (3) Tiris. The Hamada of Tindouf, where the refugee camps are located, is a barren desert plateau historically known as the "Devil's Garden." Because of the scarce rainfall and poor edaphic conditions, the Hamada is significantly poorer in forage compared with southern areas; there are few trees, no halophytic plants, and annual plants are also relatively scarce and dominated by a few species (e.g., Tetraena gaetula (Emb. \& Maire) Beier \& Thulin). The liberated territories lie south of the Hamada, with the northern sec- 


\section{Volpato \& Puri - Dormancy and Revitalization: The fate of ethnobotanical knowledge of camel forage among Sahrawi nomads and refugees}

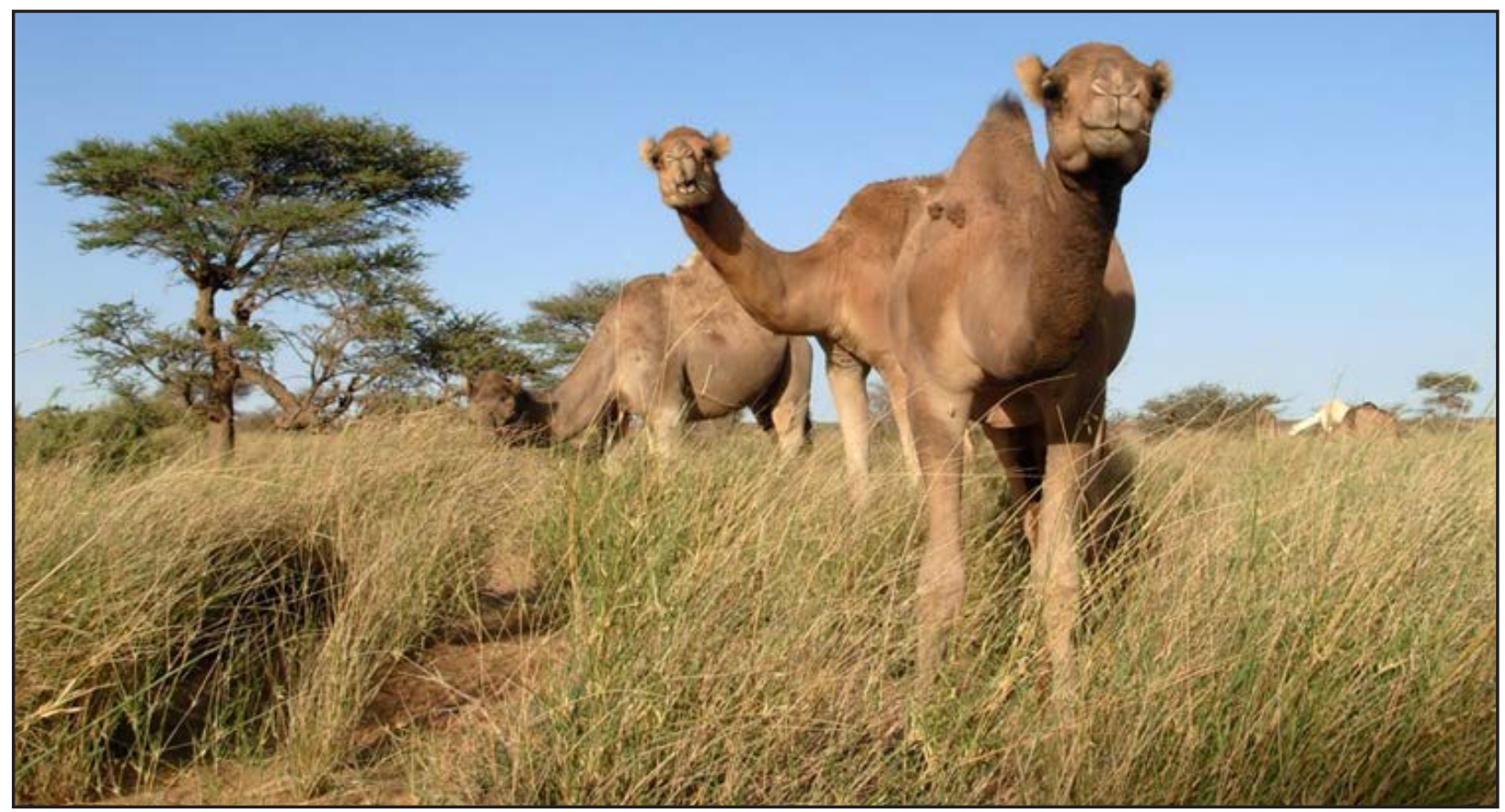

Figure 2. Acacia-Panicum vegetation in Zemmur, Western Sahara (photo by D. Rossi).

tor, called Zemmur, running east-west between the berm and northern Mauritania. Sand and gravel plains predominate, with sandstone and granite occasionally surfacing in its eastern and central zones, and with higher relief and hilly terrain in the western zone. All of Zemmur, especially the central and western areas, is gullied by inactive or occasionally active rivers that drain west into the Saguia el-Hamra, a large ephemeral river. After the rains, the Zemmur displays a savannah-like environment dominated by Acacia-Panicum vegetation (Figure 2), while flowering prairies may appear in flat gravel areas. The southern sector, known as Tiris, runs from north to south between the berm and the Mauritanian border and is more arid then Zemmur, characterized by flat sand and gravel plains from which rise characteristic black granite hills in clusters or in isolation. There are no dry riverbeds in Tiris as in Zemmur; vegetation is mostly herbaceous and adventitious, and halophytic plants cover large areas.

\section{Methodology}

The data analyzed in this paper were collected in Sahrawi refugee camps and in the Polisario-controlled liberated territories of Western Sahara between 2006 and 2009. Ethnobotanical fieldwork was carried out in accordance with standard texts (Alexiades 1996, Cunningham 2001) and also included anthropological fieldwork methods such as participant observation and interviews (Bernard 2006, Weller 1998). Interviews were conducted in Hassaniya (the Arabic language with a Berber substrate spoken by the Sahrawi) and Spanish: one local research assistant asked the questions in Hassaniya and translated the an- swers into Spanish, which is the second most frequently spoken language among the Sahrawi and which is spoken fluently by the first author, who conducted the fieldwork. To ensure that, during the interview process, no mistakes were made in translation and to clarify doubtful information, interviews were recorded and transcribed with the help of the same research assistant. Prior informed consent was obtained verbally before interviews were conducted. Participants were given an explanation of the aims, methodology, and outcomes of the study. The ethical guidelines followed were those adopted by the American Anthropological Association (1998) and the International Society of Ethnobiology (2006).

Free-listing was used to describe the Sahrawi cultural domain of martaa Ibal ("camel forage") as well as to document the distribution of associated knowledge among the Sahrawi (Borgatti 1996b, Puri 2011, Quinlan 2005, Ryan et al. 2000). The free-listing exercise was conducted with 46 Sahrawi, both nomads and refugees, who were approached directly in their tents in refugee camps or in the liberated territories. Informant sampling was purposeful in that we tried to include people across the range of sociodemographic attributes hypothesized to influence camel forage knowledge among the Sahrawi. These attributes were five: age, gender, nomadism (or not), presence/absence of camel herding at the time of the study, and total years as a nomad. In relation to this last attribute, it was found that refugees who sometimes move to the liberated territories during the rainy season (although born in the refugee camps or relocated there as children) proudly counted the time spent as seasonal nomads (often consti- 
tuted of intervals varying from a few months to a year) and provided us with a recall of the total time of these intervals, which we used for this measure. It was expected that nomads retained a wider knowledge of camel forage than refugees and that age and years of nomadic life positively influenced knowledge maintenance, too. As camel management is traditionally a male task among the Sahrawi, it was also expected that men had a greater knowledge of camel forage than women.

Free-listing generated data that both stimulated qualitative discussion about the importance of specific plants and could be used in cultural consensus analysis (CCA) and multidimensional scaling (MDS) to (1) study informant agreement and variation both within and between groups and (2) explore the causes of such knowledge distribution (Nakao \& Romney 1984, Ross et al. 2007, Weller \& Baer 2002). Follow-up semi-structured interviews were conducted with 25 of the more knowledgeable informants (selected on the basis of their free-list lengths and experience with extensive camel nomadism) to check dubious items in the lists and, more importantly, to investigate in greater detail the relevance of specific forage plants, their nomenclature, classification, ecology, and properties (e.g., in camel diet and health) as well as pathways of knowledge transmission.

The analysis of agreement and variation in free-lists using cultural domain analysis is described and justified in detail elsewhere (Puri 2011, Quinlan 2005, Weller \& Baer 2002), and several statistical software packages, including ANTHROPAC, can manage and analyze data in this way. (See Borgatti's Methods Manual (1996b) for detailed instructions for cultural domain analysis using ANTHROPAC.) The main steps can be briefly summarized. Freelists are compiled in an informant-by-item matrix, which indicates both the rank order and the presence/absence of items in each informant's list. From this, the frequency of items, their average rank in the list, and a measure of salience (based on Smith 1993) can be calculated. This is essentially a weighted average of the (inverse) rank of an item across multiple free-lists, where each list is weighted by the number of items in the list. These data indicate the contents and boundaries of the domain in question: those items that are more commonly known and/or are prototypical of the domain and thus have salience or meaning for the group, and, for some items listed infrequently, those that may not be members of the domain at all.

We can compare informants' knowledge of the domain by simply comparing the lengths of free-lists; usually, the longer the list, the more someone knows about a domain. More interesting and revealing is the variation in the items listed. Thus, we looked at the pattern of agreement in freelists for each pair of informants, measured as the percentage of items shared in their lists (a procedure known as positive matching in ANTHROPAC). An inter-informant agreement matrix of these percentages was then com- piled, which can be visually represented using Multidimensional Scaling (MDS) and/or analyzed to determine cultural consensus and intra-cultural variation.

MDS plots graphically depict the inter-informant agreement matrix, showing the relative distances between informants in a two- or three-dimensional space; those informants with similar free-lists are located closer in Cartesian space, and thus one can easily identify "groups" of informants with similar lists. Hypotheses were formed about the dimensions or variables that may underlie this distribution, and several types of regression analysis were used to test their explanatory power (Borgatti 1996b:2738, Puri 2011). In ANTHROPAC, Property Fitting (PROFIT) was used to test metric variables (age and years as a nomad), and Quadratic Assignment Procedure (QAP) was used to test non-metric variables (sex, nomadism, camel herding), as described above (see Borgatti 1996b). Our analysis provides the same results as an analysis of "residual agreement" used to determine if there are subgroups of agreement within a general consensus (Nakao \& Romney 1984, Ross et al. 2007).

Cultural Consensus Analysis (CCA), based on Romney et al. (1986) and developed as a tool in ANTHROPAC (Borgatti 1996a), applies a principle component analysis to the informants, not the items, to determine whether consensus (or shared agreement) among informants exists. It creates a model of what the consensus answer to the question(s) posed might be (in this case, what items are in the domain "camel forage") and then estimates the ability or competency of each informant to give the "culturally correct" answers. Weller and Baer (2002:11) describe CCA as follows: "In a consensus analysis, the interinformant agreement matrix is then factored by a principal axis (minimum residual) extraction method to obtain the competency scores. If the results indicate a single factor or single response pattern among the informants, then the consensus model is appropriate to describe the data." Second and third factors may also be present and often indicate either patterned intra-cultural variation such as subcultures or residual agreement. As mentioned above, we used the QAP and PROFIT tools in ANTHROPAC to test for residual agreement.

Regarding botanical methods, voucher specimens were collected with informants through a "walk in the woods" approach (Cunningham 2001) in the Hamada of Tindouf and across the liberated territories, where the first author travelled on four occasions between 2006 and 2009. Plant nomenclature follows the Sahara and Western Sahara botanical standard treatises (Dobignard et al. 1992a, 1992b, Lebrun 1998, Ozenda 1991) and the International Plant Name Index (www.ipni.org). Voucher specimens were deposited in the National Herbarium of The Netherlands (Wageningen Branch - Herbarium Vadense). 


\section{Volpato \& Puri - Dormancy and Revitalization: The fate of ethnobotanical knowledge of camel forage among Sahrawi nomads and refugees}

Qualitative data were coded and analyzed narratively (description, explanation, interpretation, quotations) using QSR NVivo. Codes were developed from the topics addressed during the interviews (e.g., classification of camel forage, organoleptic properties, and knowledge transmission), expanded into more detailed coding (e.g., specific categories of camel forage), and inductively identified and transformed into themes (e.g., knowledge revitalization). Intercoder reliability was not assessed since the data were coded by the first author alone. The software packages used to analyze quantitative data were ANTHROPAC for free-lists and Microsoft Excel® for descriptive statistics.

\section{Results}

Table 1 presents social and demographic data on the 46 Sahrawi informants-10 nomads and 36 refugees (16 of whom had never lived a nomadic life)-who were interviewed on their knowledge of camel forage. Interviewees included 12 women and 34 men, from the ages of 12 to around 70 . Ten informants herded camels, including two refugees who had been nomads and one enterprising 12-year-old boy with no past experience as a nomadic herder.

Table 2 provides a list of martaa Ibal ("camel forage") of Western Sahara according to these Sahrawi informants. About 100 plant species are reported in alphabetical order of botanical taxon, along with botanical family, voucher specimen number, local phytonym in Hassaniya, part(s) of the plant grazed, and properties attributed to the forage. About 80 botanical genera are present, the most common being Stipagrostis (4 species), Acacia, Astragalus, Euphorbia, Fagonia, Launaea, Salsola, and Tamarix (3 species each). Species belong to 32 botanical families, the most prevalent of which are Asteraceae and Fabaceae (12 species each), Amaranthaceae (11 species), Brassicaceae (8 species), and Poaceae and Zygophyllaceae (7 species).

Table 3 presents the results obtained from the ANTHROPAC analysis of the free-lists of Sahrawi informants. Informants gave a cumulative list of 83 plant names, with

Table 1. Sahrawi informant socio-demographic data, Western Sahara. Informants: (\#) numbered; Gender: (F) female, (M) male; Status: $(\mathrm{R})$ refugee, $(\mathrm{N})$ nomad; Time in years as nomad; Herds camels? (yes/no).

\begin{tabular}{|c|c|c|c|c|c|}
\hline \# & Age & Gender & Status & Time & Camels \\
\hline 1 & 63 & $M$ & $\mathrm{R}$ & 30 & no \\
\hline 2 & 29 & M & $\mathrm{R}$ & 0 & no \\
\hline 3 & 37 & $M$ & $\mathrm{R}$ & 0 & no \\
\hline 4 & 60 & M & $\mathrm{R}$ & 26 & no \\
\hline 5 & 47 & $\mathrm{~F}$ & $\mathrm{R}$ & 20 & no \\
\hline 6 & 26 & $\mathrm{~F}$ & $\mathrm{R}$ & 0 & no \\
\hline 7 & 40 & M & $\mathrm{R}$ & 5 & no \\
\hline 8 & 58 & $M$ & $\mathrm{R}$ & 32 & yes \\
\hline 9 & 68 & $M$ & $\mathrm{R}$ & 38 & no \\
\hline 10 & 47 & M & $\mathrm{R}$ & 35 & no \\
\hline 11 & 26 & $M$ & $\mathrm{R}$ & 10 & no \\
\hline 12 & 65 & $\mathrm{M}$ & $\mathrm{R}$ & 9 & no \\
\hline 13 & 28 & $M$ & $\mathrm{R}$ & 0 & no \\
\hline 14 & 25 & $\mathrm{M}$ & $\mathrm{R}$ & 5 & no \\
\hline 15 & 22 & $\mathrm{M}$ & $\mathrm{R}$ & 0 & no \\
\hline 16 & 47 & $\mathrm{M}$ & $\mathrm{R}$ & 10 & no \\
\hline 17 & 37 & $\mathrm{~F}$ & $\mathrm{R}$ & 0 & no \\
\hline 18 & 36 & $\mathrm{M}$ & $\mathrm{R}$ & 10 & no \\
\hline 19 & 34 & $M$ & $\mathrm{R}$ & 2 & no \\
\hline 20 & 39 & $\mathrm{M}$ & $\mathrm{R}$ & 0 & no \\
\hline 21 & 31 & $\mathrm{M}$ & $\mathrm{R}$ & 0 & no \\
\hline 22 & 29 & M & $\mathrm{R}$ & 2 & no \\
\hline 23 & 60 & $\mathrm{M}$ & $\mathrm{R}$ & 45 & no \\
\hline
\end{tabular}

\begin{tabular}{|c|c|c|c|c|c|}
\hline \# & Age & Gender & Status & Time & Camels \\
\hline 24 & 43 & M & $\mathrm{R}$ & 25 & yes \\
\hline 25 & 54 & M & $\mathrm{R}$ & 0 & no \\
\hline 26 & 55 & M & $\mathrm{N}$ & 55 & yes \\
\hline 27 & 28 & M & $\mathrm{R}$ & 3 & no \\
\hline 28 & 34 & $\mathrm{~F}$ & $\mathrm{R}$ & 0 & no \\
\hline 29 & 42 & $M$ & $\mathrm{R}$ & 15 & no \\
\hline 30 & 50 & $\mathrm{~F}$ & $\mathrm{R}$ & 25 & no \\
\hline 31 & 55 & $\mathrm{~F}$ & $\mathrm{R}$ & 0 & no \\
\hline 32 & 47 & $\mathrm{~F}$ & $\mathrm{R}$ & 0 & no \\
\hline 33 & 60 & $\mathrm{~F}$ & $\mathrm{R}$ & 0 & no \\
\hline 34 & 56 & M & $\mathrm{R}$ & 40 & no \\
\hline 35 & 34 & $\mathrm{~F}$ & $\mathrm{R}$ & 0 & no \\
\hline 36 & 39 & $\mathrm{~F}$ & $\mathrm{R}$ & 0 & no \\
\hline 37 & 12 & $M$ & $\mathrm{R}$ & 0 & yes \\
\hline 38 & 54 & $M$ & $\mathrm{~N}$ & 54 & yes \\
\hline 39 & 60 & M & $\mathrm{N}$ & 60 & yes \\
\hline 40 & 70 & $M$ & $\mathrm{~N}$ & 55 & no \\
\hline 41 & 40 & $\mathrm{~F}$ & $\mathrm{~N}$ & 40 & yes \\
\hline 42 & 43 & M & $\mathrm{N}$ & 30 & yes \\
\hline 43 & 50 & M & $\mathrm{N}$ & 45 & yes \\
\hline 44 & 18 & $\mathrm{~F}$ & $\mathrm{~N}$ & 18 & no \\
\hline 45 & 31 & M & $\mathrm{N}$ & 31 & no \\
\hline 46 & 58 & $M$ & $\mathrm{~N}$ & 52 & yes \\
\hline
\end{tabular}


Table 2. Forage plants grazed by camels in Western Sahara according to the Sahrawi. Parts grazed: aerial part (ap); flowers (fl); fruit (fr) ; leaves (le); shoots (sh); stems (st); young whorls (wh); young leaves (yl).

\begin{tabular}{|c|c|c|c|c|}
\hline $\begin{array}{l}\text { Species } \\
\text { (voucher number) }\end{array}$ & Family & $\begin{array}{l}\text { Hassaniya } \\
\text { phytonym }\end{array}$ & $\begin{array}{l}\text { Part(s) grazed \& } \\
\text { Hassaniya name }\end{array}$ & Properties as forage \\
\hline $\begin{array}{l}\text { Aaronsohnia pubescens } \\
\text { (Desf.) K.Bremer \& } \\
\text { Humphries (1090) }\end{array}$ & Asteraceae & Ierbien & ap & \\
\hline $\begin{array}{l}\text { Acacia ehrenbergiana } \\
\text { Hayne (1015) }\end{array}$ & Fabaceae & tamat & $\begin{array}{l}\text { le (warga tamat), } \\
\mathrm{fl} \text { (anish) }\end{array}$ & $\begin{array}{l}\text { Very good forage, with } \\
\text { properties like } A \text {. tortilis. }\end{array}$ \\
\hline $\begin{array}{l}\text { Acacia senegal (L.) Willd. } \\
\text { var. senegal (1076) }\end{array}$ & Fabaceae & amour & ap & $\begin{array}{l}\text { Good forage, with properties } \\
\text { like } A \text {. tortilis but of secondary } \\
\text { importance in Western Sahara. }\end{array}$ \\
\hline $\begin{array}{l}\text { Acacia tortilis (Forssk.) } \\
\text { Hayne subsp. } \\
\text { raddiana (Savi) } \\
\text { Brenan }(1010,1025)\end{array}$ & Fabaceae & talha & $\begin{array}{l}\text { le (warga tamat), } \\
\text { fl (anish), } \\
\text { fr (gharrub) }\end{array}$ & $\begin{array}{l}\text { Main camel forage in Western } \\
\text { Sahara, especially during } \\
\text { hot periods when trees are } \\
\text { among the few green food } \\
\text { sources; flowers are especially } \\
\text { appreciated by calves; pods } \\
\text { are also eaten and given to the } \\
\text { animals as nutraceutical. }\end{array}$ \\
\hline $\begin{array}{l}\text { Aizoon canariense } \\
\text { L. (2032) }\end{array}$ & Aizoaceae & taza & ap & Increases milk production. \\
\hline $\begin{array}{l}\text { Ammodaucus } \\
\text { leucotrichus Coss. } \\
\text { (1033) }\end{array}$ & Apiaceae & $\begin{array}{l}\text { kammuna, } \\
\text { kammunat } \\
\text { rag }\end{array}$ & ap & \\
\hline $\begin{array}{l}\text { Anabasis articulata } \\
\text { (Forssk.) Moq. }\end{array}$ & Amaranthaceae & ashram & ap & $\begin{array}{l}\text { As main forage it causes camel } \\
\text { diarrhea and possible colic, } \\
\text { but in a varied diet it is a good } \\
\text { pasture. }\end{array}$ \\
\hline $\begin{array}{l}\text { Anastatica hierochuntica } \\
\text { L. (1027) }\end{array}$ & Brassicaceae & kamsha & ap & $\begin{array}{l}\text { Very good forage when green } \\
\text { ("strong" food). }\end{array}$ \\
\hline $\begin{array}{l}\text { Androcymbium } \\
\text { punctatum Baker (2065) }\end{array}$ & Colchicaceae & $\begin{array}{l}\text { sghaa } \\
\text { t'nereb }\end{array}$ & ap, fl & \\
\hline $\begin{array}{l}\text { Anvillea radiata Coss. } \\
\text { \& Durieu (2039) }\end{array}$ & Asteraceae & negd & ap & $\begin{array}{l}\text { Good forage; it gives camel milk } \\
\text { a bitter taste. }\end{array}$ \\
\hline $\begin{array}{l}\text { Argania spinosa } \\
\text { (L.) Skeels }\end{array}$ & Sapotaceae & argan & ap & $\begin{array}{l}\text { Camels browse it, but it gives } \\
\text { milk a bitter taste; seeds (bulez) } \\
\text { accumulate in the rumen and } \\
\text { may give camels gastritis and } \\
\text { colic. }\end{array}$ \\
\hline $\begin{array}{l}\text { Asparagus } \\
\text { altissimus Munby }\end{array}$ & Asparagaceae & sekoum & ap & $\begin{array}{l}\text { Good "sweet" forage; gives milk } \\
\text { a sweet taste. } \\
\end{array}$ \\
\hline $\begin{array}{l}\text { Asphodelus tenuifolius } \\
\text { Cav. (1078) }\end{array}$ & Xanthorrhoeaceae & tazia & ap & Grazed only when dry. \\
\hline $\begin{array}{l}\text { Asteriscus graveolens } \\
\text { (Forssk.) Less. (2043) }\end{array}$ & Asteraceae & tafsa & ap & $\begin{array}{l}\text { Good forage; if grazed in big } \\
\text { amounts, it gives milk a bitter } \\
\text { taste. }\end{array}$ \\
\hline
\end{tabular}




\section{Volpato \& Puri - Dormancy and Revitalization: The fate of ethnobotanical knowledge of camel forage among Sahrawi nomads and refugees}

\begin{tabular}{|c|c|c|c|c|}
\hline $\begin{array}{l}\text { Species } \\
\text { (voucher number) }\end{array}$ & Family & $\begin{array}{l}\text { Hassaniya } \\
\text { phytonym }\end{array}$ & $\begin{array}{l}\text { Part(s) grazed \& } \\
\text { Hassaniya name }\end{array}$ & Properties as forage \\
\hline $\begin{array}{l}\text { Astragalus eremophilus } \\
\text { Boiss. (2054) }\end{array}$ & Fabaceae & umm harza & ap & $\begin{array}{l}\text { Good forage; camels like it } \\
\text { especially when in flower; } \\
\text { when pastured alone or in main } \\
\text { quantities it gives a strong and } \\
\text { unpleasant taste to camel tripe } \\
\text { that then must be washed more } \\
\text { before cooking. }\end{array}$ \\
\hline $\begin{array}{l}\text { Astragalus mareoticus } \\
\text { Delile }\end{array}$ & Fabaceae & umm harza & $\mathrm{ap}, \mathrm{fl}$ & $\begin{array}{l}\text { The flowers especially are an } \\
\text { appreciated camel forage. }\end{array}$ \\
\hline $\begin{array}{l}\text { Astragalus vogelii } \\
\text { (Webb) Bornm. }\end{array}$ & Fabaceae & ter & ap & $\begin{array}{l}\text { Excellent forage when green, } \\
\text { it increases and qualitatively } \\
\text { improves milk production, } \\
\text { fattens camels, and gives to } \\
\text { camel milk an appreciated } \\
\text { taste. The ingestion of many } \\
\text { seeds from dry plants can give } \\
\text { camels an intoxication known } \\
\text { as fenter. }\end{array}$ \\
\hline Atractylis aristata Batt. & Asteraceae & sherb jmel & ap & $\begin{array}{l}\text { Fattening forage; "it gives a big } \\
\text { hump to camels that feed from } \\
\text { it." }\end{array}$ \\
\hline $\begin{array}{l}\text { Atriplex halimus L. } \\
\text { (1052) }\end{array}$ & Amaranthaceae & legtaf & ap & $\begin{array}{l}\text { Important salty forage, it } \\
\text { improves the quality of the milk, } \\
\text { giving it a salty taste; it may give } \\
\text { nervous problems to camels } \\
\text { that eat it in huge quantities } \\
\text { when there are no other forages } \\
\text { available. }\end{array}$ \\
\hline $\begin{array}{l}\text { Balanites aegyptiacus } \\
\text { (L.) Delile (1086) }\end{array}$ & Zygophyllaceae & teichat & ap & \\
\hline Calendula sp. & Asteraceae & gahuan & ap & \\
\hline $\begin{array}{l}\text { Calligonum comosum } \\
\text { L'Hér. }\end{array}$ & Polygonaceae & awrash & ap & $\begin{array}{l}\text { Good forage when in flower and } \\
\text { fruit. }\end{array}$ \\
\hline $\begin{array}{l}\text { Capparis decidua } \\
\text { (Forssk.) Edgew. }\end{array}$ & Capparaceae & ighnin & ap, fr (bujrali) & $\begin{array}{l}\text { Watery forage important in the } \\
\text { summer period for the water it } \\
\text { provides. }\end{array}$ \\
\hline $\begin{array}{l}\text { Caylusea hexagyna } \\
\text { M.L. Green (2068) }\end{array}$ & Resedaceae & dhenban & ap & Good pasture. \\
\hline $\begin{array}{l}\text { Convolvulus trabutianus } \\
\text { Schweinf. \& Muschl. }\end{array}$ & Convolvulaceae & gandul & ap & Forage appreciated by camels. \\
\hline $\begin{array}{l}\text { Cornulaca } \\
\text { monacantha Delile }\end{array}$ & Amaranthaceae & had & ap & $\begin{array}{l}\text { Important forage, especially } \\
\text { during the summer and during } \\
\text { droughts. }\end{array}$ \\
\hline $\begin{array}{l}\text { Cotula cinerea } \\
\text { Delile (1083) }\end{array}$ & Asteraceae & $\begin{array}{l}\text { rebruba } \\
\text { (without } \\
\text { flowers), } \\
\text { gartufa } \\
\text { (with } \\
\text { flowers) }\end{array}$ & $\mathrm{ap}, \mathrm{fl}$ & $\begin{array}{l}\text { It gives a bitter and not } \\
\text { appreciated taste to camel milk. }\end{array}$ \\
\hline
\end{tabular}




\begin{tabular}{|c|c|c|c|c|}
\hline $\begin{array}{l}\text { Species } \\
\text { (voucher number) }\end{array}$ & Family & $\begin{array}{l}\text { Hassaniya } \\
\text { phytonym }\end{array}$ & $\begin{array}{l}\text { Part(s) grazed \& } \\
\text { Hassaniya name }\end{array}$ & Properties as forage \\
\hline $\begin{array}{l}\text { Crotalaria saharae } \\
\text { Coss. }(2060)\end{array}$ & Fabaceae & fula & ap & $\begin{array}{l}\text { Good forage; when eaten in } \\
\text { abundant quantities a camel } \\
\text { sweats a lot and may give signs } \\
\text { of rmah (craziness) and its milk } \\
\text { has a strong appreciated smell } \\
\text { of fula flowers. }\end{array}$ \\
\hline $\begin{array}{l}\text { Cullen plicata } \\
\text { (Delile) C.H.Stirt }\end{array}$ & Fabaceae & totrat & ap & Very good forage. \\
\hline $\begin{array}{l}\text { Cymbopogon } \\
\text { schoenanthus (L.) } \\
\text { Spreng. (1087) }\end{array}$ & Poaceae & $\begin{array}{l}\text { idkhir, } \\
\text { liedjir }\end{array}$ & ap & \\
\hline $\begin{array}{l}\text { Cynodon dactylon } \\
\text { (L.) Pers. }\end{array}$ & Poaceae & nijm & ap & \\
\hline $\begin{array}{l}\text { Cyperus conglomeratus } \\
\text { Rottb. }\end{array}$ & Cyperaceae & sad & ap & $\begin{array}{l}\text { Good forage; it increases milk } \\
\text { production. }\end{array}$ \\
\hline $\begin{array}{l}\text { Diplotaxis pitardiana } \\
\text { Maire (2055) }\end{array}$ & Brassicaceae & $\begin{array}{l}\text { deid han } \\
\text { (without } \\
\text { flower), } \\
\text { karkas (with } \\
\text { flowers, } \\
\text { flower beds) }\end{array}$ & ap & $\begin{array}{l}\text { Good forage; it gives a strong } \\
\text { floral smell to the milk of } \\
\text { pasturing camels, at times } \\
\text { causing calves to reduce or stop } \\
\text { milking. }\end{array}$ \\
\hline $\begin{array}{l}\text { Echium horridum } \\
\text { Batt. (1091) }\end{array}$ & Boraginaceae & harsha & ap & Sweet forage. \\
\hline $\begin{array}{l}\text { Ephedra alata } \\
\text { Decne. (1067) }\end{array}$ & Ephedraceae & $\begin{array}{l}\text { shdida, } \\
\text { alenda }\end{array}$ & ap & $\begin{array}{l}\text { Grazed only if better forages are } \\
\text { absent. }\end{array}$ \\
\hline $\begin{array}{l}\text { Eremobium aegyptiacum } \\
\text { (Spreng.) Asch. ex Boiss. }\end{array}$ & Brassicaceae & shgaa & ap & $\begin{array}{l}\text { Good forage, but it gives an } \\
\text { unpleasant taste to camel meat } \\
\text { and milk. }\end{array}$ \\
\hline $\begin{array}{l}\text { Euphorbia } \\
\text { balsamifera Aiton }\end{array}$ & Euphorbiaceae & fernan & ap & $\begin{array}{l}\text { Grazed when green, but it can } \\
\text { give camels digestive troubles. }\end{array}$ \\
\hline $\begin{array}{l}\text { Euphorbia calyptrata } \\
\text { Coss. \& Kralik (2063) }\end{array}$ & Euphorbiaceae & rammadah & ap & $\begin{array}{l}\text { Forage not much appreciated; } \\
\text { when the camel touches the } \\
\text { plant, its milk can cause eye } \\
\text { irritation; grazed only by camels } \\
\text { that are not used to the area. }\end{array}$ \\
\hline $\begin{array}{l}\text { Euphorbia granulata } \\
\text { Forssk. (1055) }\end{array}$ & Euphorbiaceae & $\begin{array}{l}\text { kbidet } \\
\text { ed-dab }\end{array}$ & ap & $\begin{array}{l}\text { Not much appreciated pasture } \\
\text { by camels; grazed only by } \\
\text { camels that are not used to the } \\
\text { area. }\end{array}$ \\
\hline Fagonia glutinosa Delile & Zygophyllaceae & ledesma & ap & Important forage. \\
\hline Fagonia jolyi Batt. & Zygophyllaceae & tleha & ap & $\begin{array}{l}\text { Good forage when green in } \\
\text { spring. }\end{array}$ \\
\hline Fagonia sp. & Zygophyllaceae & daaishe & ap & $\begin{array}{l}\text { Not much appreciated by } \\
\text { camels. }\end{array}$ \\
\hline Farsetia aegyptia Turra & Brassicaceae & zaaza & ap & Pasture appreciated by camels. \\
\hline Farsetia stylosa R.Br. & Brassicaceae & $\begin{array}{l}\text { akshir, el } \\
\text { gadhm }\end{array}$ & ap & $\begin{array}{l}\text { Good forage especially in } \\
\text { combination with askaf. }\end{array}$ \\
\hline $\begin{array}{l}\text { Glossonema boveanum } \\
\text { (Decne.) Decne. }\end{array}$ & Apocynaceae & ashakan & ap & \\
\hline
\end{tabular}


Volpato \& Puri - Dormancy and Revitalization: The fate of ethnobotanical knowledge of camel forage among Sahrawi nomads and refugees

\begin{tabular}{|c|c|c|c|c|}
\hline $\begin{array}{l}\text { Species } \\
\text { (voucher number) }\end{array}$ & Family & $\begin{array}{l}\text { Hassaniya } \\
\text { phytonym }\end{array}$ & $\begin{array}{l}\text { Part(s) grazed \& } \\
\text { Hassaniya name }\end{array}$ & Properties as forage \\
\hline $\begin{array}{l}\text { Grewia tenax } \\
\text { (Forssk.) Fiori }\end{array}$ & Malvaceae & leghlia & ap, yl & \\
\hline $\begin{array}{l}\text { Gymnocarpos decandrus } \\
\text { Forssk. (1093) }\end{array}$ & Caryophyllaceae & shifne & ap & $\begin{array}{l}\text { Important salty forage; it gives } \\
\text { milk a salty taste. }\end{array}$ \\
\hline $\begin{array}{l}\text { Haloxylon scoparium } \\
\text { Pomel (1021) }\end{array}$ & Amaranthaceae & remth & ap & $\begin{array}{l}\text { Grazed by camels only when no } \\
\text { other forage is available. }\end{array}$ \\
\hline $\begin{array}{l}\text { Helianthemum lippii (L.) } \\
\text { Dum. Cours. (1034) }\end{array}$ & Cistaceae & erghig & ap & Grazed when green. \\
\hline $\begin{array}{l}\text { Heliotropium crispum } \\
\text { Desf. (2053) }\end{array}$ & Boraginaceae & lehbaliya & ap & Good camel forage. \\
\hline $\begin{array}{l}\text { Ifloga spicata (Forssk.) } \\
\text { Sch.Bip. (2033) }\end{array}$ & Asteraceae & asb el-abd & ap & \\
\hline $\begin{array}{l}\text { Kleinia anteuphorbium } \\
\text { (L.) Haw. }\end{array}$ & Asteraceae & shbarto & ap & $\begin{array}{l}\text { Good forage when green as } \\
\text { well as when dried. }\end{array}$ \\
\hline $\begin{array}{l}\text { Launaea arborescens } \\
\text { (Batt.) Murb. (1071) }\end{array}$ & Asteraceae & umm Ibena & ap & $\begin{array}{l}\text { When green, it gives to camel } \\
\text { meat and milk a bitter and lightly } \\
\text { hot taste. }\end{array}$ \\
\hline $\begin{array}{l}\text { Launaea mucronata } \\
\text { (Forssk.) Muschl. (2034) }\end{array}$ & Asteraceae & el mekker & ap & $\begin{array}{l}\text { Good forage when mixed with } \\
\text { others, but it gives to camel } \\
\text { meat and milk an unpleasant } \\
\text { bitter taste; the name of the } \\
\text { plant means "the bitter one." }\end{array}$ \\
\hline $\begin{array}{l}\text { Launaea nudicaulis (L.) } \\
\text { Hook.f. (2027) }\end{array}$ & Asteraceae & gherrema & ap & Sweet forage. \\
\hline Limonium spp. & Plumbaginaceae & garza & $\begin{array}{l}\text { le, fl, flower } \\
\text { beds (azatim) }\end{array}$ & $\begin{array}{l}\text { Good forage; flower beds are } \\
\text { very appreciated by camels. }\end{array}$ \\
\hline Lotus spp. (2025) & Fabaceae & atig & ap & $\begin{array}{l}\text { Good and fattening forage; } \\
\text { increases and improves milk } \\
\text { production; other informants } \\
\text { say it gives an unpleasant and } \\
\text { strong taste to camel milk. }\end{array}$ \\
\hline $\begin{array}{l}\text { Lupinus digitatus } \\
\text { Forssk. (2058) }\end{array}$ & Fabaceae & $\begin{array}{l}\text { umm } \\
\text { el-feifat, } \\
\text { boutreisira }\end{array}$ & ap & \\
\hline $\begin{array}{l}\text { Lycium intricatum Boiss. } \\
\text { (1085) }\end{array}$ & Solanaceae & ghardeg & ap & Good and "strong" forage. \\
\hline $\begin{array}{l}\text { Maerua crassifolia } \\
\text { Forssk. }(1007,1048)\end{array}$ & Capparaceae & atil & $\begin{array}{l}\text { le (sadra el } \\
\text { hadra) }\end{array}$ & $\begin{array}{l}\text { Important forage; the flower is } \\
\text { especially appreciated. }\end{array}$ \\
\hline $\begin{array}{l}\text { Mesembryanthemum } \\
\text { cryptanthum Hook.f. }\end{array}$ & Aizoaceae & afzu & ap & Good forage. \\
\hline $\begin{array}{l}\text { Moltkiopsis ciliata } \\
\text { (Forssk.) I.M.Johnst. }\end{array}$ & Boraginaceae & nshal & ap & Appreciated by camels. \\
\hline $\begin{array}{l}\text { Monsonia nivea (Decne.) } \\
\text { Decne. ex Webb }\end{array}$ & Geraniaceae & rekum & ap & $\begin{array}{l}\text { Salty forage; when camels eat } \\
\text { it alone and not in variation with } \\
\text { other pastures, it gives colic and } \\
\text { diarrhea that disappears after } \\
\text { few days. }\end{array}$ \\
\hline
\end{tabular}




\begin{tabular}{|c|c|c|c|c|}
\hline $\begin{array}{l}\text { Species } \\
\text { (voucher number) }\end{array}$ & Family & $\begin{array}{l}\text { Hassaniya } \\
\text { phytonym }\end{array}$ & $\begin{array}{l}\text { Part(s) grazed \& } \\
\text { Hassaniya name }\end{array}$ & Properties as forage \\
\hline $\begin{array}{l}\text { Morettia canescens } \\
\text { Boiss. (2045) }\end{array}$ & Brassicaceae & tebazuaga & ap & $\begin{array}{l}\text { It increases milk production in } \\
\text { camels and makes milk more } \\
\text { nutritious; it is also appreciated } \\
\text { by camels when dry. }\end{array}$ \\
\hline Neurada procumbens L. & Rosaceae & saadan & ap & Good forage when green. \\
\hline $\begin{array}{l}\text { Nitraria retusa } \\
\text { (Forssk.) Asch. }\end{array}$ & Zygophyllaceae & gherzim & ap, fl (akawar) & $\begin{array}{l}\text { Good forage, especially young } \\
\text { leaves and flowers. }\end{array}$ \\
\hline $\begin{array}{l}\text { Nucularia perrinii } \\
\text { Batt. (1047) }\end{array}$ & Amaranthaceae & askaf & ap & $\begin{array}{l}\text { Best and "strongest" forage for } \\
\text { camels in Western Sahara and } \\
\text { most important salty plant; "it } \\
\text { gives salts, strength, and mass } \\
\text { to camels" and nutraceutical } \\
\text { properties to the milk. }\end{array}$ \\
\hline $\begin{array}{l}\text { Panicum turgidum } \\
\text { Forssk. (1051) }\end{array}$ & Poaceae & $\begin{array}{l}\text { mrokba, } \\
\text { umm rekba }\end{array}$ & ap & Good forage. \\
\hline $\begin{array}{l}\text { Patellifolia procumbens } \\
\text { (C.Sm.) A.J.Scott, } \\
\text { Ford-Lloyd \& } \\
\text { J.T.Williams (1075) }\end{array}$ & Amaranthaceae & silk & ap & $\begin{array}{l}\text { Watery forage not regarded as } \\
\text { very good but important during } \\
\text { the summer period. }\end{array}$ \\
\hline Pergularia tomentosa $\mathrm{L}$. & Apocynaceae & $\begin{array}{l}\text { ghalqa, } \\
\text { umm el-jlud }\end{array}$ & ap & $\begin{array}{l}\text { Camels sometimes graze it } \\
\text { when it is dry and in absence of } \\
\text { other pastures. }\end{array}$ \\
\hline Periploca laevigata Aiton & Apocynaceae & hallab & ap & $\begin{array}{l}\text { Important sweet forage; drinking } \\
\text { milk from a camel that grazed } \\
\text { this plant is believed to protect } \\
\text { from illness and to strengthen } \\
\text { children. }\end{array}$ \\
\hline Picris asplenioides L. & Asteraceae & uden naja & ap & $\begin{array}{l}\text { Sweet forage that gives to milk } \\
\text { a sweet taste. }\end{array}$ \\
\hline $\begin{array}{l}\text { Reseda villosa } \\
\text { Coss. (1072) } \\
\end{array}$ & Resedaceae & yamin & ap & $\begin{array}{l}\text { Good forage, but it gives an } \\
\text { unpleasant smell to camel milk. }\end{array}$ \\
\hline $\begin{array}{l}\text { Retama raetam } \\
\text { (Forssk.) Webb }\end{array}$ & Fabaceae & rtam & $\mathrm{fl}, \mathrm{fr}$ & $\begin{array}{l}\text { Camels graze only flowers and } \\
\text { fruits; stems are eaten only } \\
\text { when there are no other forages } \\
\text { and in exceptional cases; it } \\
\text { gives to milk a slightly sour or } \\
\text { bitter taste. }\end{array}$ \\
\hline Rumex vesicarius $\mathrm{L}$. & Polygonaceae & hmued & ap & $\begin{array}{l}\text { Camels graze it, but it is } \\
\text { considered by Sahrawi a sour } \\
\text { forage of low quality. }\end{array}$ \\
\hline $\begin{array}{l}\text { Salsola imbricata Forssk. } \\
\text { (1054) }\end{array}$ & Amaranthaceae & ghasal & ap & $\begin{array}{l}\text { Camels graze it only in drought } \\
\text { periods; it can cause colic and } \\
\text { diarrhea; for this it is regarded } \\
\text { as a strong and acid plant able } \\
\text { to treat intestinal parasites } \\
\text { through the diarrhea caused. }\end{array}$ \\
\hline Salsola longifolia Forssk. & Amaranthaceae & laasal & ap & Important forage, rich in water. \\
\hline
\end{tabular}




\section{Volpato \& Puri - Dormancy and Revitalization: The fate of ethnobotanical knowledge of camel forage among Sahrawi nomads and refugees}

\begin{tabular}{|c|c|c|c|c|}
\hline $\begin{array}{l}\text { Species } \\
\text { (voucher number) }\end{array}$ & Family & $\begin{array}{l}\text { Hassaniya } \\
\text { phytonym }\end{array}$ & $\begin{array}{l}\text { Part(s) grazed \& } \\
\text { Hassaniya name }\end{array}$ & Properties as forage \\
\hline $\begin{array}{l}\text { Salsola tetrandra Forssk. } \\
(2020)\end{array}$ & Amaranthaceae & laarad & ap & $\begin{array}{l}\text { Important salty forage; if eaten } \\
\text { alone or in huge quantities can } \\
\text { cause diarrhea; it eliminates } \\
\text { intestinal parasites because it is } \\
\text { an acid plant; it gives camel milk } \\
\text { a salty taste. }\end{array}$ \\
\hline $\begin{array}{l}\text { Salvia aegyptiaca L. } \\
\text { (1049) }\end{array}$ & Lamiaceae & tezouknit & ap & Grazed when green. \\
\hline $\begin{array}{l}\text { Schouwia thebaica } \\
\text { Webb. }\end{array}$ & Brassicaceae & yerdhir & ap & $\begin{array}{l}\text { Appreciated forage, but it gives } \\
\text { to camel milk a very bitter taste. }\end{array}$ \\
\hline $\begin{array}{l}\text { Sclerocephalus arabicus } \\
\text { (Decne.) Boiss. (2031) }\end{array}$ & Caryophyllaceae & $\begin{array}{l}\text { shamra, } \\
\text { tamra }\end{array}$ & ap & \\
\hline $\begin{array}{l}\text { Searsia albida } \\
\text { (Schousb.) Moffett }\end{array}$ & Anacardiaceae & zauaya & ap, fr (anafis) & \\
\hline $\begin{array}{l}\text { Searsia tripartita } \\
\text { (Ucria) Moffett (1023) }\end{array}$ & Anacardiaceae & shdari & le & $\begin{array}{l}\text { Important forage especially in } \\
\text { periods of drought. }\end{array}$ \\
\hline $\begin{array}{l}\text { Stipagrostis acutiflora } \\
\text { (Trin. \& Rupr.) De Winter }\end{array}$ & Poaceae & $\begin{array}{l}\text { aserdum } \\
\text { (when } \\
\text { green), sfar } \\
\text { (when dry) }\end{array}$ & ap & Good forage. \\
\hline $\begin{array}{l}\text { Stipagrostis ciliata } \\
\text { (Desf.) De Winter }\end{array}$ & Poaceae & $\begin{array}{l}\text { ataf (when } \\
\text { green), } \\
\text { zigzig } \\
\text { (when dry) }\end{array}$ & ap & $\begin{array}{l}\text { Good and appreciated forage, } \\
\text { especially when dry. }\end{array}$ \\
\hline $\begin{array}{l}\text { Stipagrostis plumosa } \\
\text { Munro ex T.Anderson } \\
(2030)\end{array}$ & Poaceae & nsil & ap & $\begin{array}{l}\text { Very important forage when } \\
\text { green as well as dry; the best } \\
\text { forage during summer. }\end{array}$ \\
\hline $\begin{array}{l}\text { Stipagrostis pungens } \\
\text { (Desf.) De Winter }\end{array}$ & Poaceae & $\begin{array}{l}\text { sbat, el } \\
\text { halfa (when } \\
\text { dry), ilig } \\
\text { (flowers } \\
\text { and seeds) }\end{array}$ & ap & $\begin{array}{l}\text { Important forage especially } \\
\text { during periods of drought due } \\
\text { to the resistance of the plant; } \\
\text { camels eat only the dry plant } \\
\text { and the flowers. }\end{array}$ \\
\hline $\begin{array}{l}\text { Suaeda vermiculata } \\
\text { Forssk. ex J.F.Gmel. } \\
(1082)\end{array}$ & Amaranthaceae & sueid & ap & $\begin{array}{l}\text { Salty forage but not as good } \\
\text { as the others in the category; } \\
\text { when eaten it paints black the } \\
\text { lips and teeth of the camels and } \\
\text { sometimes causes colic, thus } \\
\text { they graze it only in absence of } \\
\text { better forages. }\end{array}$ \\
\hline $\begin{array}{l}\text { Tamarix amplexicaulis } \\
\text { Ehrenb. }\end{array}$ & Tamaricaceae & fersig & ap & \\
\hline $\begin{array}{l}\text { Tamarix aphylla (L.) } \\
\text { H. Karst. }\end{array}$ & Tamaricaceae & lezl & ap & \\
\hline Tamarix gallica L. (1063) & Tamaricaceae & tarfa & ap & $\begin{array}{l}\text { Flowers and young stems are } \\
\text { appreciated by camels; it is not } \\
\text { regarded as an important or } \\
\text { nutritious forage. }\end{array}$ \\
\hline
\end{tabular}




\begin{tabular}{|c|c|c|c|c|}
\hline $\begin{array}{l}\text { Species } \\
\text { (voucher number) }\end{array}$ & Family & $\begin{array}{l}\text { Hassaniya } \\
\text { phytonym }\end{array}$ & $\begin{array}{l}\text { Part(s) grazed \& } \\
\text { Hassaniya name }\end{array}$ & Properties as forage \\
\hline $\begin{array}{l}\text { Tetraena gaetula } \\
\text { (Emb. \& Marie) Beier } \\
\text { \& Thulin (1065) }\end{array}$ & Zygophyllaceae & aggaya & ap & $\begin{array}{l}\text { When green, it is a strong and } \\
\text { acid forage, giving a salty taste } \\
\text { to camel milk; during summer, } \\
\text { it is a pasture for "leisure" for } \\
\text { camels because it is rich in } \\
\text { water. }\end{array}$ \\
\hline $\begin{array}{l}\text { Tetraena simplex (L.) } \\
\text { Beier \& Thulin }\end{array}$ & Zygophyllaceae & lemuelha & ap & It gives milk a bitter taste. \\
\hline $\begin{array}{l}\text { Teucrium chardonianum } \\
\text { Maire \& Wilczek }\end{array}$ & Lamiaceae & $\begin{array}{l}\text { sadra } \\
\text { el-beida }\end{array}$ & ap & Good forage. \\
\hline $\begin{array}{l}\text { Traganum nudatum } \\
\text { Delile }\end{array}$ & Amaranthaceae & damran & ap & $\begin{array}{l}\text { Important forage; if eaten in } \\
\text { huge quantities and not mixed } \\
\text { with other pastures, may cause } \\
\text { diarrhea due to its salt content. }\end{array}$ \\
\hline Trichodesma spp. (2048) & Boraginaceae & $\begin{array}{l}\text { harsha } \\
\text { haibe }\end{array}$ & ap & Good forage. \\
\hline Trigonella anguina Delile & Fabaceae & el gard & ap, se & $\begin{array}{l}\text { Complementary forage for } \\
\text { camels when green, but it is a } \\
\text { very strong and toxic pasture } \\
\text { when with seeds; camels that } \\
\text { eat many seeds will "explode" } \\
\text { with colic and strong meteorism; } \\
\text { when in flower, these give a } \\
\text { strong smell to the milk. }\end{array}$ \\
\hline Zilla spinosa (L.) Prantl. & Brassicaceae & shobrak & ap & Good forage. \\
\hline $\begin{array}{l}\text { Ziziphus lotus (L.) Lam. } \\
\text { (1002) }\end{array}$ & Rhamnaceae & sdir & ap & \\
\hline n.d. & & $\begin{array}{l}\text { musran } \\
\text { lehuar }\end{array}$ & ap & Good forage. \\
\hline
\end{tabular}

Table 3. Results of ANTHROPAC analysis of camel forage free-lists by Sahrawi informants, Western Sahara. *Plant included in the cultural consensus model.

\begin{tabular}{|l|l|r|r|}
\hline Item & Botanical taxon & $\begin{array}{r}\text { Frequency (\%) } \\
\text { N = 46 }\end{array}$ & $\begin{array}{r}\text { Smith's } \\
\text { Salience Index }\end{array}$ \\
\hline Talha * & Acacia tortilis (Forssk.) Hayne & $45(97.8)$ & 0.752 \\
\hline Askaf * & Nucularia perrinii Batt. & $35(76)$ & 0.666 \\
\hline Ter * & Astragalus vogelii (Webb) Bornm. & $30(65.2)$ & 0.405 \\
\hline Mrokba * & Panicum turgidum Forssk. & $28(60.8)$ & 0.406 \\
\hline Nsil * & Stipagrostis plumosa Munro ex T.Anderson & $27(58.7)$ & 0.411 \\
\hline Mharza & Astragalus eremophilus Boiss. & $25(54.3)$ & 0.305 \\
\hline Sbat & Stipagrostis pungens (Desf.) De Winter & $23(50)$ & 0.356 \\
\hline Lehbaliya & Heliotropium crispum Desf. & $22(47.8)$ & 0.26 \\
\hline Tamat & Acacia ehrenbergiana Hayne & $21(45.6)$ & 0.303 \\
\hline Tafsa & Asteriscus graveolens (Forssk.) Less. & $20(43.5)$ & 0.226 \\
\hline Karkas & Diplotaxis pitardiana Maire & $19(41.3)$ & 0.227 \\
\hline Laarad & Salsola tetrandra Forssk. & & 0.304 \\
\hline Ghassel & Salsola imbricata Forssk. & 0.232 \\
\hline
\end{tabular}



knowledge of camel forage among Sahrawi nomads and refugees

\begin{tabular}{|c|c|c|c|}
\hline Item & Botanical taxon & $\begin{array}{r}\text { Frequency (\%) } \\
N=46\end{array}$ & $\begin{array}{r}\text { Smith's } \\
\text { Salience Index }\end{array}$ \\
\hline Gartufa & Cotula cinerea Delile & $17(37)$ & 0.148 \\
\hline Negd & Anvillea radiata Coss. \& Durieu & \multirow[t]{4}{*}{$16(34.8)$} & 0.161 \\
\hline Sadan & Neurada procumbens $\mathrm{L}$. & & 0.174 \\
\hline Aserdum & Stipagrostis acutiflora (Trin. \& Rupr.) De Winter & & 0.173 \\
\hline Damran & Traganum nudatum Delile & & 0.206 \\
\hline El Had & Cornulaca monacantha Delile & $15(32.6)$ & 0.235 \\
\hline Ghardeq & Lycium intricatum Boiss. & $14(30.4)$ & 0.172 \\
\hline Garza & Limonium spp. & \multirow[t]{2}{*}{$13(28.2)$} & 0.093 \\
\hline Fula & Crotalaria saharae Coss. & & 0.137 \\
\hline Ledesme & Fagonia glutinosa Delile & $12(26)$ & 0.077 \\
\hline Kamsha & Anastatica hierochuntica L. & \multirow[t]{2}{*}{$11(24)$} & 0.06 \\
\hline Harsha & Echium horridum Batt. & & 0.106 \\
\hline Remth & Haloxylon scoparium Pomel & \multirow[t]{2}{*}{$10(21.7)$} & 0.085 \\
\hline Shdary & Searsia tripartita (Ucria) Moffett & & 0.135 \\
\hline Lierguig & Helianthemum lippii (L.) Dum.Cours. & \multirow[t]{5}{*}{$9(19.5)$} & 0.074 \\
\hline Atil & Maerua crassifolia Forssk. & & 0.077 \\
\hline Yerdhir & Schouwia thebaica Webb. & & 0.055 \\
\hline Ataf & Stipagrostis ciliata (Desf.) De Winter & & 0.097 \\
\hline Aggaya & Tetraena gaetula (Emb. \& Marie) Beier \& Thulin & & 0.125 \\
\hline Lerbien & Aaronsohnia pubescens (Desf.) K.Bremer \& Humphries & \multirow[t]{5}{*}{$8(17.4)$} & 0.046 \\
\hline Ashram & Anabasis articulata (Forssk.) Moq. & & 0.108 \\
\hline Dhenban & Caylusea hexagyna M.L.Green & & 0.056 \\
\hline Zaaza & Farsetia aegyptia Turra & & 0.073 \\
\hline Atig & Lotus spp. & & 0.065 \\
\hline Sekum & Asparagus altissimus Munby & \multirow[t]{5}{*}{$7(15.2)$} & 0.047 \\
\hline Legtaf & Atriplex halimus L. & & 0.086 \\
\hline Gandul & Convolvulus trabutianus Schweinf. \& Muschl. & & 0.057 \\
\hline Totrat & Cullen plicata (Delile) C.H.Stirt & & 0.067 \\
\hline Tebazuaga & Morettia canescens Boiss. & & 0.069 \\
\hline Liedkhir & Cymbopogon schoenanthus (L.) Spreng. & \multirow[t]{4}{*}{$6(13)$} & 0.026 \\
\hline Sghaa & Eremobium aegyptiacum (Spreng.) Asch. ex Boiss. & & 0.033 \\
\hline Hallaba & Periploca laevigata Aiton & & 0.043 \\
\hline Liedmin & Reseda villosa Coss. & & 0.05 \\
\hline Tleha & Fagonia jolyi Batt. & \multirow[t]{6}{*}{$5(10.8)$} & 0.025 \\
\hline Akshir & Farsetia sp. & & 0.036 \\
\hline Butreisira & Lupinus digitatus Forssk. & & 0.043 \\
\hline Rekum & Monsonia nivea (Decne.) Decne. ex Webb & & 0.045 \\
\hline Gherzim & Nitraria retusa (Forssk.) Asch. & & 0.051 \\
\hline Hmued & Rumex vesicarius $\mathrm{L}$. & & 0.051 \\
\hline Amour & Acacia senegal (L.) Willd. & \multirow[t]{2}{*}{$4(8.7)$} & 0.033 \\
\hline Ashakan & Glossonema boveanum (Decne.) Decne. & & 0.011 \\
\hline
\end{tabular}




\begin{tabular}{|c|c|c|c|}
\hline Item & Botanical taxon & $\begin{array}{r}\text { Frequency (\%) } \\
N=46\end{array}$ & $\begin{array}{r}\text { Smith's } \\
\text { Salience Index }\end{array}$ \\
\hline Shifne & Gymnocarpos decandrus Forssk. & \multirow[t]{5}{*}{4 (8.7) cont. } & 0.023 \\
\hline Nshal & Moltkiopsis ciliata (Forssk.) I.M.Johnst. & & 0.056 \\
\hline Laasal & Salsola longifolia Forssk. & & 0.04 \\
\hline Sueid & Suaeda vermiculata Forssk. ex J.F.Gmel. & & 0.05 \\
\hline Fersig & Tamarix amplexicaulis Ehrenb. & & 0.025 \\
\hline Umm Lbena & Launaea arborescens (Batt.) Murb. & \multirow[t]{7}{*}{$3(6.5)$} & 0.036 \\
\hline Mekker & Launaea mucronata (Forssk.) Muschl. & & 0.022 \\
\hline Tezoukenit & Salvia aegyptiaca L. & & 0.011 \\
\hline Tamra & Sclerocephalus arabicus (Decne.) Boiss. & & 0.021 \\
\hline Tarfa & Tamarix gallica $\mathrm{L}$. & & 0.046 \\
\hline Shobrak & Zilla spinosa (L.) Prantl & & 0.009 \\
\hline Sdir & Ziziphus lotus (L.) Lam. & & 0.014 \\
\hline Tazia & Asphodelus tenuifolius Cav. & \multirow[t]{5}{*}{$2(4.3)$} & 0.021 \\
\hline Awrash & Calligonum comosum L'Hér. & & 0.005 \\
\hline Ighnin & Capparis decidua (Forssk.) Edgew. & & 0.024 \\
\hline Legliya & Grewia tenax (Forssk.) Fiori & & 0.009 \\
\hline Uden Naja & Picris asplenioides $\mathrm{L}$. & & 0.003 \\
\hline Taza & Aizoon canariense L. & \multirow[t]{12}{*}{$1(2.2)$} & 0.014 \\
\hline Argan & Argania spinosa (L.) Skeels & & 0.009 \\
\hline Gahuan & Calendula sp. & & 0.002 \\
\hline Sad & Cyperus conglomeratus Rottb. & & 0.013 \\
\hline Daaishe & Fagonia sp. & & 0.001 \\
\hline Shbarto & Kleinia anteuphorbium (L.) Haw. & & 0.011 \\
\hline Gherema & Launaea nudicaulis (L.) Hook.f. & & 0.002 \\
\hline Henna & Lawsonia inermis L. & & 0.016 \\
\hline Umm Halluz & Lotus jolyi Batt. & & 0.011 \\
\hline Afzou & Mesembryanthemum cryptanthum Hook.f. & & 0.011 \\
\hline Rtem & Retama raetam (Forssk.) Webb & & 0.006 \\
\hline Lezl & Tamarix aphylla (L.) H.Karst. & & 0.001 \\
\hline
\end{tabular}

individual free-lists ranging from 1 to 34 and an average length per free-list of 17 plants. The plants cited are reported in order of their frequency of mention (i.e., how many informants mentioned a plant), along with their botanical taxon, Hassaniya name, and Smith's Salience Index. Talha, Acacia tortilis (Forssk.) Hayne, the umbrella thorn tree, was mentioned by all but one informant and had the highest overall salience index. The halophytic askaf, Nucularia perrinii Batt., was the second most salient plant listed, mentioned by $75 \%$ of informants. Eight plants were mentioned by more than half of all informants, and 18 were mentioned by more than a third, which both confirms the existence of the domain and demonstrates a core of shared knowledge about its contents. Table 3 also shows the five plants that form the cultural consensus model of shared knowledge of the domain as calculated by ANTHROPAC. This model represents those items of the domain most likely to be mentioned by a typical member of the sampled population, both because they were mentioned more frequently and also sooner (i.e., has a higher rank) in informants' lists.

Table 4 presents results obtained from ANTHROPAC's consensus analysis of the forage free-lists (Borgatti 1996b:39). The consensus model included the five most salient plants listed, thus informants showed a high degree of agreement (or competency) with the cultural consensus (average competency $=71 \%$ ). The first factor explained $83 \%$ of the variation (eigenvalue $=24.74$ ), the second factor $13 \%$ (eigenvalue $=3.86$ ), and the third factor 


\section{Volpato \& Puri - Dormancy and Revitalization: The fate of ethnobotanical knowledge of camel forage among Sahrawi nomads and refugees}

$4 \%$ (eigenvalue $=1.22$ ). Since the ratio of the first factor to the second is greater than 3 , this indicates high overall agreement among informants, indicating that they belong to a single culture (i.e., share knowledge of camel forage). The second factor suggests enough residual agreement to indicate subgroups, or clusters of agreement, within the study population (Nakao \& Romney 1984, Ross et al. 2007).

Table 4. Consensus analysis results for Sahrawi free-lists on camel forage. The average competency score was $0.717(\mathrm{SD}=0.153)$.

\begin{tabular}{|c|c|c|c|}
\hline Factor & Eigenvalue & $\%$ & Ratio \\
\hline 1 & 24.74 & 83 & 6.414 \\
\hline 2 & 3.86 & 12.9 & 3.161 \\
\hline 3 & 1.22 & 4.1 & \\
\hline & 29.82 & 100 & \\
\hline
\end{tabular}

To visualize the individual differences in Sahrawi free-lists and begin to explore subgroups of agreement and explanatory variables, a non-metric multidimensional scaling (MDS) analysis was performed on the inter-informant agreement matrix in ANTHROPAC (Figure 3). The plot shows similarity among informants' free-lists as distance in two-dimensional space, where those with more similar lists are closer and those more dissimilar are further apart (Borgatti 1996b:33).

The distribution shows a grouping of informants in the lower left quadrant, with the rest evenly distributed above and to the upper right of the plot, and only a few outliers. This group contains nomads and older refugees who once were nomadic (at least until 1975), as well as Polisario soldiers who have spent long periods in Western Sahara in contact with nomads or have been themselves herders (e.g., informant 34), and young refugees who took up seasonal nomadism over the past decade (e.g., informant 27). In the results of a PROFIT analysis of hypothesized explanatory variables (Table 5 below), the vector on the MDS plot shows the highest values for years as a nomad in this lower quadrant $\left(R^{2}=0.56, p<0.001\right)$, which is also very much correlated with the length of free-lists. Age, not shown above, is also correlated with years as a nomad, but because even younger nomads know more than refugees who are older than them, age explains less of the variation $\left(R^{2}=0.32, p<0.002\right)$. The difference among men and women was small but significant $\left(R^{2}=0.027, p\right.$ $<0.046)$, while status as refugee did not matter $(p<0.39)$ since most were former nomads, many of whom are now elderly and have retained knowledge equivalent to that of the permanent nomads interviewed (refugees with no nomadic experience had dramatically shorter free-lists). For the same reasons, owning or herding camels also did

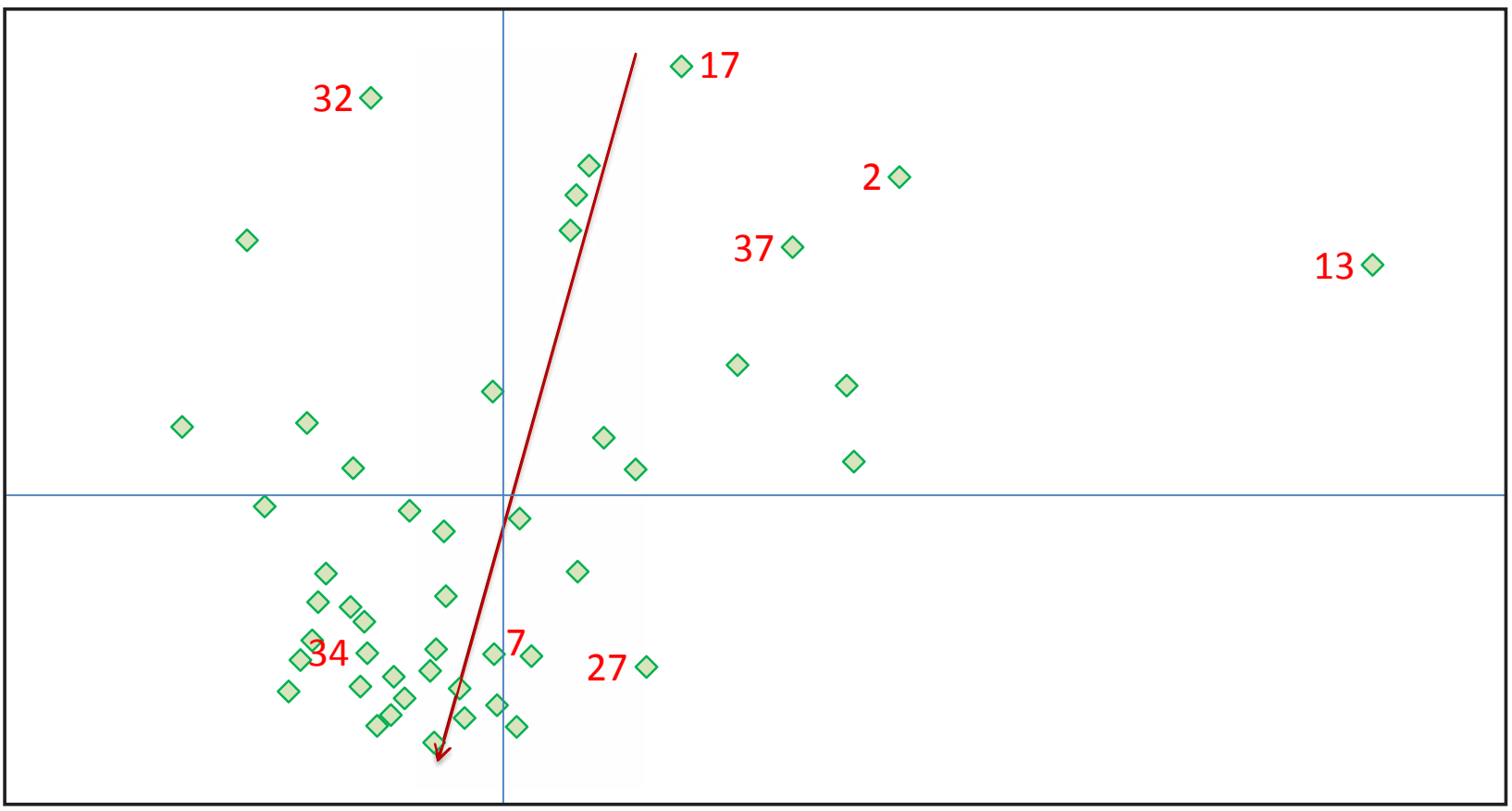

Figure 3. Non-metric multidimensional scaling of Sahrawi's similarity in naming camel forage plants in the Western Sahara region. Each diamond on the map represents a Sahrawi informant; those with similar free-lists are relatively closer in space. Only informants (diamonds) who are referred to in the text are numbered in the graph. Axes, in themselves, have no meaningful value and orientation is arbitrary; the only thing that counts is the relative distance between points. The vector represents the variable Years as nomad regressed on MDS plot using the PROFIT program in ANTHROPAC, where value increases from top to bottom (see Table 5). 
not make a difference $(p<0.33)$ in knowledge, but it was noted that three of the 36 refugees now herd camels and there is an expectation that more may do so in the future (Table 5). One more informant (informant 7) had attempted to re-initiate camel husbandry about three years prior to the interview, but the she-camel that he bought had died. He said, "I will soon get a stronger she-camel. I'll have her reproduced, and her milk will be precious for my old parents." Younger nomads and less-experienced refugees are scattered somewhat evenly across the MDS plot, showing some knowledge and variation in their shorter lists. Informant 13 is a noticeable outlier on the right side of the graph who listed a single plant, Tetraena gaetula. He is a relatively young refugee ( 28 years old) without any experience of nomadism or other forms of engagement with Western Sahara territory. Tetraena is in fact not a good camel forage, nor part of the cultural consensus model; nevertheless, it is the most common species growing in the surroundings of the refugee camps that was often listed by refugees as camel forage since it is one of the few plants present in the Hamada of Tindouf and thus known to refugees through direct experience. Informant 2 has very similar attributes to informant 13 and also listed T. gaetula, as well as Acacia tortilis. The situation is not different with informant 17 , who only listed $A$. tortilis, and informant 32, who listed four species including $A$. tortilis; both are refugees with no nomadic experience, who are 37 and 47 years old, respectively. Informant 37 is a 12 -year-old boy who was born and has lived all his life in the El Aaiún refugee camp and who owns two she-camels with their calves. Despite his short free-list of plants present around the camp (e.g., T. gaetula, Tamarix gallica L., Anvillea radiata Coss. et Durieu) as well as $A$. tortilis, he is, like many more boys and young adults in the refugee camps, interested in acquiring more herding knowledge, an exemplar of the desire to revitalize livelihoods and lifestyles once thought to be forever lost.

Table 5. Summary statistics from PROFIT and QAP analysis of hypothesized explanatory variables about Sahrawi from Western Sahara. *Significant at $\alpha=0.05$; ${ }^{* *}$ Significant at $\alpha=0.001$.

\begin{tabular}{|l|l|l|}
\hline Variable & $\boldsymbol{R}^{\mathbf{2}}$ & $\boldsymbol{p}$-value \\
\hline Age & 0.323 & $0.002^{*}$ \\
\hline Sex & 0.027 & $0.046^{*}$ \\
\hline Group (refugee or nomad) & 0.004 & 0.39 \\
\hline Ever lived nomadic & 0.406 & $0.0001^{* *}$ \\
\hline Years spent as nomad & 0.561 & $0.001^{* *}$ \\
\hline Present camel herding & 0.004 & 0.33 \\
\hline
\end{tabular}

\section{Discussion}

To summarize, our analysis of the free-lists pertaining to the domain of camel forage indicates that nomadic experience and, to some extent, age are the most impor- tant factors underlying the differences among informants. Interestingly, men and women differ little in their knowledge scores (more on this below). While we expected refugees to have less knowledge than nomads, this is clearly only the case for those who were raised in refugee camps. Despite being unable to herd camels for such a long time, elder refugees have not lost their knowledge of camel forage, and the fact that there is a broad consensus among the entire study population of the most salient plants means that some knowledge is being passed on to younger refugees, but certainly not as much as might be. Whether this holds into the future is of interest both to us, as researchers interested in knowledge transmission and transformation, and to older Sahrawi, who are concerned about the loss of camel herding, knowledge, meaning, and identity that is evident all around them in refugee camps. As one informant put it, "We are people of the desert. We know the desert, its plants, its animals, its landscapes; we know our camels. A refugee camp is no place for us!"

\section{Forage species in the cultural consensus model}

According to the results of the cultural consensus analysis, five species (i.e., Acacia tortilis, Nucularia perrinii, Astragalus vogelii (Webb) Bornm., Panicum turgidum Forssk., and Stipagrostis plumosa Munro ex T.Anderson) make up the cultural consensus model; they are the most salient plants in the domain, and most Sahrawi are likely to list them as camel forage. Indeed, according to Sahrawi herders, these particular plants are the most important camel forage in Western Sahara. Below, each of these species and their congenerics are briefly discussed in terms of their ecology and use.

Acacias are important forage trees in arid and semi-arid areas of Africa (Wickens 1995) as well as Western Sahara (Gauthier-Pilters 1961). In the consensus analysis, Acacia tortilis was the camel forage that the Sahrawi most often cited (97.8\%). Acacia ehrenbergiana Hayne was cited by 22 informants (47.8\%) and Acacia senegal (L.) Willd. by four $(8.7 \%)$, this order well representing the relative presence and distribution of the three species in Western Sahara. Acacia tortilis (talha) is by far the most common tree species, growing to $10 \mathrm{~m}$ in height and occupying the dry riverbeds of the Zemmur. Talha is of utmost importance to livestock as well as to humans; the Sahrawi use its parts and derived products in food, medicine, construction, and veterinary medicine (Barrera et al. 2007, Volpato et al. 2012). Talha especially is the main browse for camels in Western Sahara during the autumnal months, before and just after the first autumn rains, similar to neighboring Saharan areas (Gauthier-Pilters 1961). During the cold season, it is reported that camels favor acacia because then it bears fruit. In fact, acacia pods (garrub) are said to be nutraceutical, able "to fatten camels rapidly," so the Sahrawi and the Moors of Mauritania collect and feed them to camels (Leriche 1953). 


\section{Volpato \& Puri - Dormancy and Revitalization: The fate of ethnobotanical knowledge of camel forage among Sahrawi nomads and refugees}

Nucularia perrinii (askaf) is the second most salient plant, mentioned by 35 informants $(76 \%)$. There is very little information in the literature about this small halophytic shrub of the Amaranthaceae; there are no studies about its ecology, its current geographical distribution, or its chemical content, and it is usually cited only in ethnographic accounts about Sahrawi tribes and in regional plant lists (Boyer 1962, Caro Baroja 1955, Guinea 1948, Lebrun 1998, Ozenda 1991). The plant is endemic to Western and Central Sahara and is known to Arabic-speaking pastoralists as askaf (or âskâf, āskāf; Ould Mohamed Baba 2006) and to Tamasheq-speaking Tuareg as tassak (Benchelah et al. 2000). Askaf reaches its maximum diffusion in the rocky plains of Western Sahara, particularly in Tiris where it is the dominant "salt" species and may form monospecific populations (Correra 2006, Guinea 1945). Sahrawi herders widely acknowledge that askaf is resistant to drought and is a crucial source of salts and water for camels (Figure 4). Indeed, it is considered as a panacea: "Camels don't fall sick if there is askaf to graze, and they recover from an illness if they graze askaf," one informant explained; it is "the most curative and important forage, which gives strength and stamina to camels," according to another. More than a half-century ago, observers recorded that askaf played a fundamental role in camel diets in Western Sahara, especially in the cold season after the rains and before annuals had sprouted (Caro Baroja 1955, Doménech 1946).
In this period, camels switch to the "cure of hatba" (i.e., "salt cure"), a diet based on askaf and other halophytic plants, which "cleans camels' blood" due to its purgative effect and gives to camel meat a red and firm texture and a taste of askaf (Boyer 1962).

Astragalus vogelii (ter) is a very common annual herb in the inland plains of Western Sahara and is the third most salient taxa cited by 30 informants $(65.2 \%)$. A congeneric, Astragalus eremophilus Boiss. (mharza), is sixth, cited by 25 informants (54.3\%). Astragalus vogelii is considered to be the most important green forage for camels in Tiris, where it becomes very abundant in some areas after rains, forming prairies over many kilometers. It is reported that camels grazing green ter have increased milk production and weight gain.

The fourth and fifth plants that make up the consensus model are two grasses, Panicum turgidum (mrokba) and Stipagrostis plumosa (nsil), which are sometimes respectively referred to as the "king and queen" of camel forage. The clumping bunchgrass $P$. turgidum, along with Acacia tortilis, form the Acacia-Panicum association characteristic of the dry riverbeds of the Zemmur, where they are the most common species. In contrast, S. plumosa is characteristic of Tiris, where it grows on minimal sand deposits and may form prairies after rainfall. Stipagrostis plumosa was cited by 27 informants, Stipagrostis pungens (Desf.)

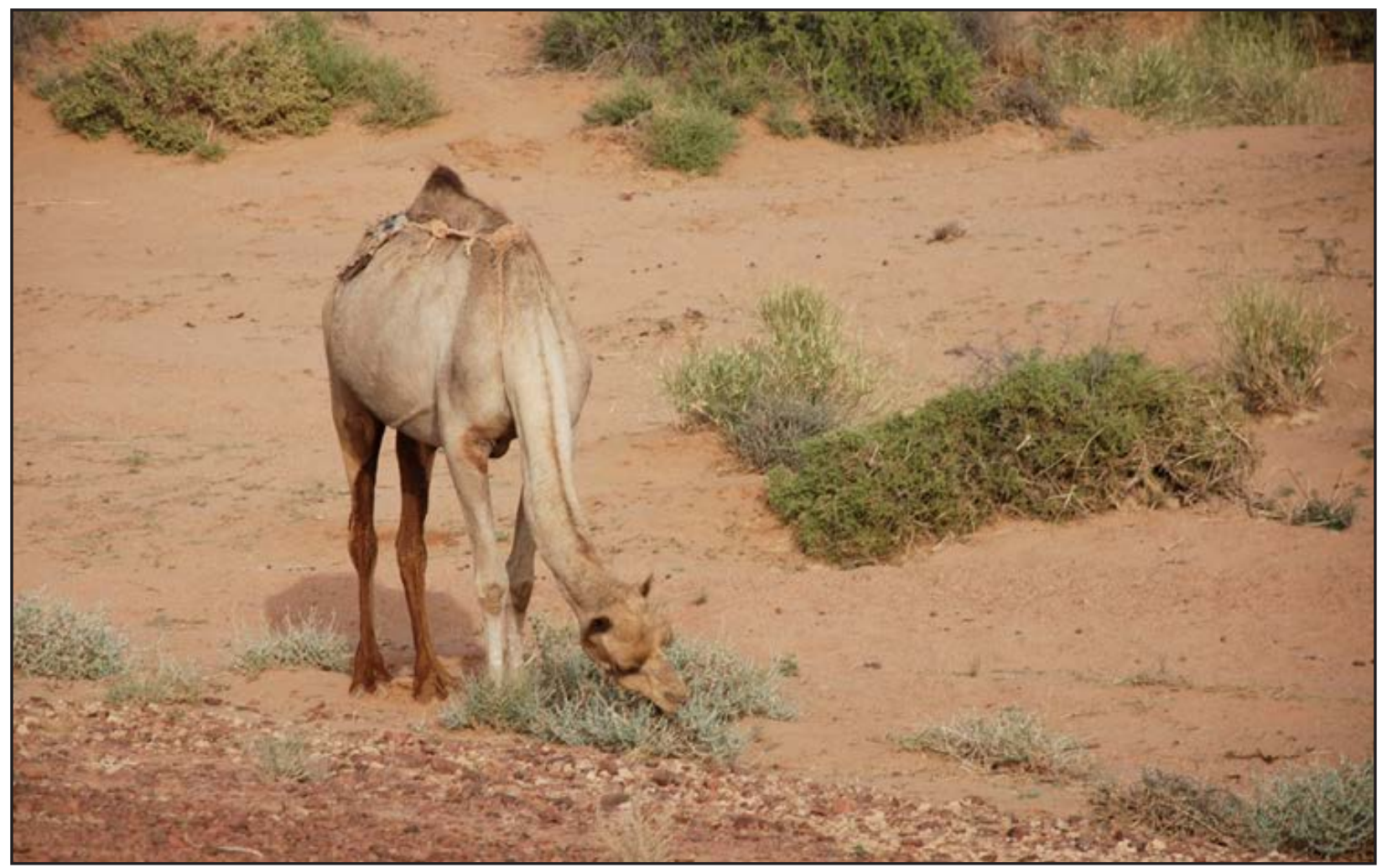

Figure 4. A camel grazes a plant of Nucularia perrinii Batt. (askaf) in Zemmur, Western Sahara (photo by G. Volpato). 
De Winter by 25, Stipagrostis acutiflora (Trin. et Rupr.) De Winter by 16, and Stipagrostis ciliata (Desf.) de Winter by nine informants. These are important camel forage species in sandy areas of south and east Western Sahara (e.g., there are vast populations of S. pungens in the sandy region of Mijek).

\section{Folk classification of camel forage in Sahrawi culture}

The Sahrawi classify camel forage into a series of functional and morphological categories that were investigated through semi-structured interviews. The classification of camel forage by the Sahrawi is schematically represented in Figure 5. Each of the plants that form part of the cultural consensus model is prototypical of high ranking (or more inclusive) Sahrawi plant life form categories (Berlin 1992): Acacia tortilis of sdar (trees), Nucularia perrinii of hatba (halophytic plants), Astragalus vogelii of both rbiya (annual herbs) and shmide (dry annual herbs), and Panicum turgidum and Stipagrostis plumosa of leshish (grasses). Below, each of these categories is briefly discussed, starting with the categories of rbiya and sdar, which appear to divide sadra, the unique beginner equivalent of "plant." Sadra (plant) is the singular of sdar (trees) in Hassaniya, while in Arabic the generic term plant is nabaat. Thus, the Sahrawi use the same term to indicate trees and plants in general. The term rbiya indicates annual plants "that grow with the rain" (i.e., herbs), whereas sdar, in contrast, defines "all those plants that do not come out with the rain" (i.e., trees and bushes; see also Ould Mohamed Baba 2006). Sdar is further divided into two main sub-categories: sdar (trees) and hatba (or summa), which includes all other non-annual plants, most of which are halophytic bushes. Summa largely overlaps with hatba, although the former is used to address plants in general terms, while the latter is used in reference to camel forage.

Forty-three percent of 100 species reported in Table 1 and 35 out of $83(42 \%)$ species reported in the consensus list are rbiya. A large share of rbiya species consists equally of Fabaceae (e.g., Astragalus spp., Crotalaria saharae Coss., Lotus spp.) and Asteraceae (e.g., Aaronsohnia pubescens (Desf.) K.Bremer \& Humphries, Cotula cinerea Delile, Picris asplenioides L.). Other rbiya are Heliotropium crispum Desf., Diplotaxis pitardiana Maire, Limonium spp. (Figure 6), Echium horridum Batt., and Helianthemum lippii (L.) Dum. Cours. Rbiya are annual herbs when green and mature (i.e., with flowers or with the green parts fully developed); alternatively, the sprouting first leaves from the cotyledon that appear soon after rains are known as zerrea while shoots are called zad'f, and both are considered to be too weak to be grazed. Dobignard et al. (1992a) define rébia as "temporary vegetation appearing after the rains, constituted essentially by ephemerophytes, but that may include also some hemicryptophytes and geophytes" and further note that this category corresponds to that of acheb used in East Morocco and South Algeria. These new herbs are important to camels since they are especially high in nutrients and low in less-digestible fibers (Dobignard et al. 1992a). In Western Sahara, they take from one to three months to complete their reproductive cycle and produce new seeds; after that, with the increasing late spring temperature, they dry quickly and become a camel food resource known to the Sahrawi as shmide.

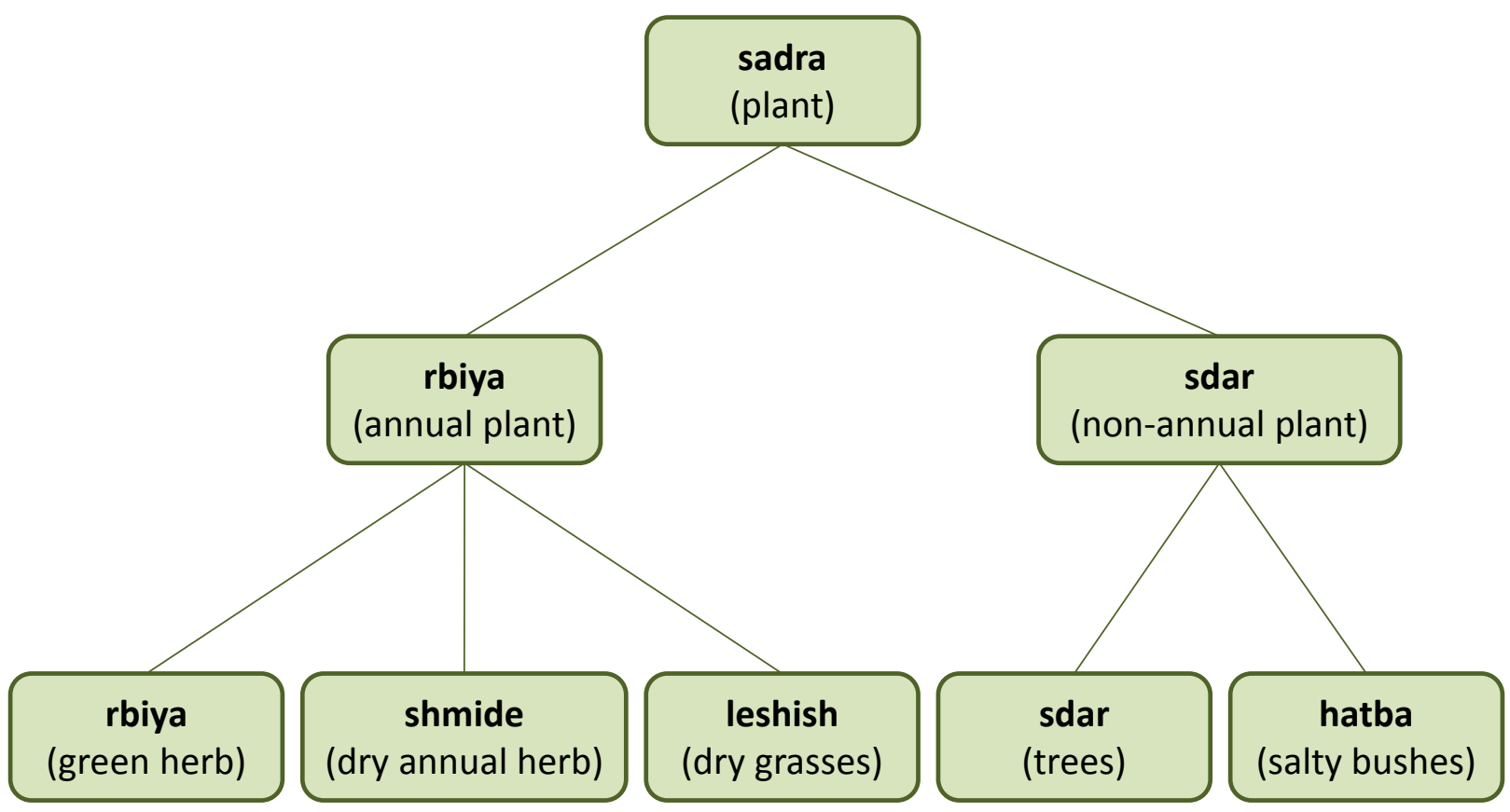

Figure 5. Sahrawi classification of camel forage in Western Sahara region. 


\section{Volpato \& Puri - Dormancy and Revitalization: The fate of ethnobotanical knowledge of camel forage among Sahrawi nomads and refugees}

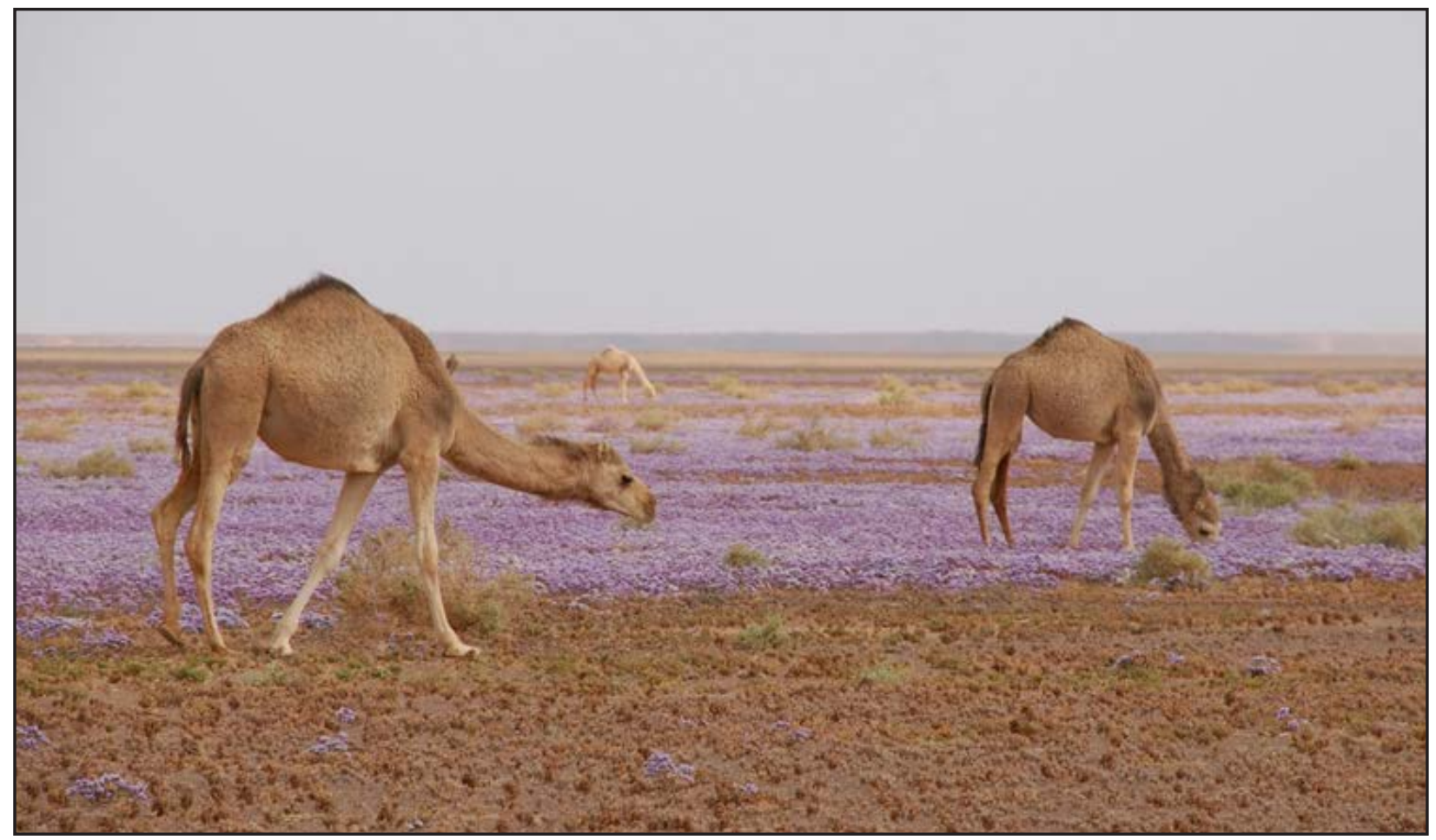

Figure 6. Camels grazing on a prairie of Limonium species (azatim) in Mehris (photo by D. Rossi).

The category of shmide includes all those rbiya that grow after the rains of the previous winter and are present in dry form during the summer and up to a year after rains. Thus, shmide may include dry populations of Cotula cinerea, Limonium spp., or Diplotaxis pitardiana, among others. Shmide is considered to be rich in salts and nutrients and an important resource for camels during summers and through the following winter in the absence of new rains. In the annals of Sahrawi tribes there are years called "the year of shmide" due to its abundance following the previous year's heavy rains (Caro Baroja 1955). Some herders also state that a very light winter rain may be worse than no rain at all, as it is not enough to stimulate the growth of rbiya, and at the same time it washes out salts and nutrients from shmide, reducing its quality as a forage. Likewise, although spring and early summer rains may regenerate part of the green forage (new rbiya), they are sometimes regarded as harmful to the quality of shmide, as they "roast" the plants (Boyer 1962). In the words of one informant, "While there is no rain, shmide will at least fill and sustain the camels."

Besides shmide, the Sahrawi distinguish another kind of dry forage: leshish. Leshish ("that can be cut") refers to dry annual and perennial grasses (e.g., Panicum turgidum, Stipagrostis spp.) and is regarded as an important camel food source, especially during summer and as a last source of fodder during droughts. In the words of two Sahrawi, "Without rains, camels may feed for years from leshish," and "Leshish and plenty of water are enough for camels during the hottest months." The term comes from the fact that these plants can be cut, harvested, and fed to camels as fodder. The importance and specific function of dry grasses for camels is not only stressed by their inclusion in a specific category, but also by the use of different taxonomic terms to contrast the green ("without seed") and dry ("with seed") individuals of the same species. Stipagrostis species, for example, are described as drought-resistant and of particular importance to camels when they are dry. Given the differential importance of Stipagrostis species as a forage when green or dry, the Sahrawi identify them with two different phytonyms (e.g., aserdum versus sfar, ataf versus zigzig, sbat versus elhalfa), according to their status. It is reported that camels never eat $S$. pungens when it is green but only when it is dry. In the past, in spring, camels were brought to a "cure of ilig"-the dry inflorescence of $S$. pungens. During this cure, which lasted about three weeks, camels would gain strength and become prepared for the heat of the summer (Boyer 1962).

Shmide and leshish are important especially throughout summer, whereas in late summer and autumn (before the rains and the growth of rbiya) herds rely largely on trees (sdar), which include 16 species in Table 1 (e.g., Acacia spp., Maerua crassifolia Forssk., Searsia spp., Tamarix spp.), and on salty and drought-resistant plants (hatba) of the Amaranthaceae (i.e., Nucularia perrinii, Salsola spp., 
Traganum nudatum Delile, Cornulaca monacantha Delile). Thirteen species in Table 1 are salty forage plants belonging to the category hatba, all belong to the Amaranthaceae except for Lycium intricatum Boiss. (Solanaceae) and Monsonia nivea (Decne.) Decne. ex Webb (Geraniaceae). According to informants, the most important member of hatba is $N$. perrinii. After that, at least half of the informants listed Salsola tetrandra Forssk. and Salsola imbricata Forssk., Traganum nudatum, Cornulaca monacantha, and Lycium intricatum. Notably, populations of these different species preferentially occupy different areas of Western Sahara: N. perrinii grows in the extended rocky steppes of the interior, $C$. monacantha grows in sandy soils and dune areas, $S$. tetrandra is predominant in some areas of Zemmur, and Atriplex halimus L. grows along the coast (Dobignard et al. 1992a). In Sahrawi camel dietary terms, hatba plants are said to be "like meat for humans," food that "makes camels grow." According to informants, in good years where different forage plants are available, camels rely largely on rbiya and hatba, feeding alternatively from plants from these categories and grazing on hatba plants preferentially in the mornings or the late afternoons.

It is known from other studies that camels graze often and preferentially on salty (halophytic) species, especially on plants with fleshy leaves that are characteristically high in protein and low in fiber content and which have the advantage of developing green aerial parts during both dry and wet periods (Gauthier-Pilters \& Dagg 1981, Wilson 1989). In fact, to absorb and store water, camels need about six to eight times as much salt as other herbivores; consequently, halophytic plants can contribute up to one third of the total diet of camels in the Sahara, with an even larger share during dry seasons and periods of drought (Gauthier-Pilters \& Dagg 1981). Because of their need for salts, Sahrawi herders brought camels to "salt cures" of about ten days in December, January, or February and sometimes in spring (April, May), after having grazed on rbiya. Similarly, it is known that other African nomadic pastoralists (e.g., Moors, Tuareg, and Somali) take camels annually to a "salt cure" (Correra 2006, Farah et al. 2004, Farid 1989).

As the above discussion indicates, the specific categories of camel forage recognized by the Sahrawi play different roles in camel nutrition throughout the year. Following the analysis of Boyer (1962:46) for the Reguibat tribe, that of Gauthier-Pilters (1961) for nomads in northwestern Sahara, and our own data and observations in the field, four nomadic seasons and respective patterns of camel dietary preferences among Sahrawi nomads can be distinguished: (1) a winter rainy season (early-December to late-February), when "camels fatten" by grazing on rbiya as well as other green forages of sdar and hatba; (2) spring (early-March to early-June), when camels progressively move from a diet based on green plants to one based on dry plants (shmide and leshish), with the "cure of ilig" (the inflorescence of Stipagrostis pungens) in between; (3) summer (early-June to early-September), when camels rely heavily on dry forage and on drought-resistant hatba plants; and (4) autumn, when camels rely largely on hatba and sdar before rains "green" the desert again.

The data show that a vast folk knowledge about the forage plants of Western Sahara (i.e., morphology, ecology, properties) is embedded in Sahrawi folk classification. This knowledge is essential to the patterns and cycles of movement of Sahrawi nomads and their herds and to the optimal exploitation of Western Saharan foraging resources, which are scarce both in time and space. The classification system also reveals much about the nomadic practices that embed and are embedded in Sahrawi ethnobotanical knowledge and that form the basis of the Sahrawi's complex adaptive management system. In fact, as other authors note in reference to patterns of nomadism and seasonal migration in the Sahara and the Sahel (Berkes et al. 2000, Niamir-Fuller 1998), annual cycles of livestock movement to different pasture areas and forage resources provide a rotational management system that enables the production and reproduction of livestock (and of human societies based on that livestock) and aims at a rational use of forage resources and the recovery of heavily grazed areas. This knowledge is thus crucial to the survival of Sahrawi nomads engaging in extensive husbandry and should be seen as foundational knowledge for refugees that seek a path toward independence and recovery of lost nomadic livelihoods through seasonal nomadism.

\section{Forage and camel milk and meat}

Because camel milk was the main output of camel husbandry and a staple food in the Sahrawi pastoral system, it is not surprising that the Sahrawi recognize in detail the relations between forage and the taste, smell, or health and nutritional properties of camel milk. The taste of milk is particularly influenced by the plants that milking camels graze, and some plants transmit their characteristic (pleasant or not) smell and flavor to camel milk. Of the 100 plants reported in Table 1, Sahrawi informants recognized a relation between 25 of them and the organoleptic or medicinal/nutraceutical properties of camel milk. Aizoon canariense L., Astragalus vogelii, Cyperus conglomeratus Rottb., Lotus spp., and Morettia canescens Boiss.- the latter being very abundant in Western Sahara plains soon after the first rains-are regarded as galactogen plants (i.e., they increase the production of milk of the she-camels grazing them), whereas Atriplex halimus and Periploga laevigata Aiton, among others, are believed to improve the milk's nutraceutical properties. Generally speaking, camel milk is believed to retain the medicinal properties of the plants that she-camels graze.

Most of the relations reported between forage and camel milk are related to the latter's organoleptic properties. The Sahrawi distinguish the taste given to camel milk by 


\section{Volpato \& Puri - Dormancy and Revitalization: The fate of ethnobotanical knowledge of camel forage among Sahrawi nomads and refugees}

sweet, salty, watery, and bitter forage. In normal grazing conditions, camels move from grazing one kind of species to another in accordance with their needs (GauthierPilters \& Dagg 1981). The taste and smell of the plants grazed in each area and season is then reflected in the taste and smell of milk. The taste and smell of the milk, in turn, tells the nomad which plants the camels have grazed and, given the different relative presence of forage plants across nomadic circuits, also where the camel has grazed. Through this process, the taste and smell of camel milk become representative of and attached to customary grazing areas, and hence also become an element of cultural identity. For example, Sahrawi nomads perceived that the "perfect" light-salty taste of Nucularia perrinii in camel milk (and meat) is characteristic of Western Sahara, in contrast with other nomads (e.g., Tuareg), their territory (where the plant is absent), and the taste of the milk. The Sahrawi also appreciate the sweet taste given to camel milk by "sweet forage" such as Asparagus altissimus Munby and $P$. asplenioides. They also praise the smell given by flowers of Astragalus vogelii and Crotalaria saharae. However, strong flower perfumes in camel milk may also have negative consequences; if a camel consumes too much Diplotaxis pitardiana which, after rains, forms yellow flower beds of many square-kilometers in Zemmur, calves may stop suckling due to the strong smell of the plant in the mother's milk. Finally, several plants (e.g., Anvillea radiata, Asteriscus graveolens Forssk., Launaea spp., Reseda villosa Coss.), especially when grazed in large quantities and/or with little variation in species, are reported to give camel milk a bitter (other times defined as "heavy" or "unpleasant") taste, which the Sahrawi do not appreciate at all. Another plant that gives camel milk a bitter and heavy taste if consumed in abundance is Schowia thebaica Webb; according to Gast et al. (1969), it confers a taste similar to that of cabbage. According to Sahrawi informants, drinking milk with a strong bitter taste imparted by these plants may cause a folk illness called eghindi, a health condition characterized by somatic reactions with a variety of symptoms (e.g., skin rashes, burning sensations, weakness, edema) due to exposure to several environmental agents (e.g., smells) or dietary sources (e.g., salty or bitter foods, burned meat) (Volpato \& Waldstein 2014).

Camel meat's organoleptic, nutritional, and medicinal properties are also influenced by the plants that camels consume. For example, the tripe of a camel fed largely from Astragalus eremophilus is said to have a strong and unpleasant taste, which requires them to be washed more. Similarly, Launaea mucronata Muschl. is called el mekker ("the bitter one") and passes an unpleasant bitter taste to camel meat. Boyer (1962:37) also discusses variation in camel meat's taste related to forage among the Reguibat: salty pastures are reported to give meat a "natural seasoning," N. perrinii a "rubbery" texture and "herbal" taste, and the presence of Cotula cinerea in pastures gives the meat a "minty" taste.
Knowledge about forage plants that influence the taste and properties of camel milk and meat indicates one way in which cultural values underpin local knowledge systems. Sahrawi practices around the taste and smell of camel products are not only the result of cumulative personal preferences; they are embedded in Sahrawi cultural values and identity. In these tastes and smells, the Sahrawi recognize those of their (lost and partly recovered) homeland and of their customary nomadic territories and further associate such tastes and smells with concepts such as freedom (e.g., crossing the desert with camels, accessing their homeland) and belonging (e.g., to a group of people with specific food practices and preferences).

\section{Distribution and transmission of knowledge about camel forage among contemporary Sahrawi}

Results from consensus and PROFIT analysis performed on camel forage free-lists have shown that forage knowledge is unevenly distributed within the Sahrawi study population and that this distribution can be explained by nomadic experience and age as the most important underlying factors. Thus, elderly refugees, who were once camel herders, retained much of their knowledge of forage, knowledge that is comparable to that of present-day nomads. However, as might be expected, the disengagement of most Sahrawi refugees from camels and nomadism as a consequence of forced displacement and sedentarization has led among younger refugees to a lack of opportunity and need for learning about forage. Moreover, decades of prohibition of access to nomadic territories meant a loss of the setting where forage knowledge was typically transmitted in the past. Even in the refugee camps, past pathways of transmission that might have conveyed some of the theoretical knowledge of the elders to the young have been broken or ignored for a variety of reasons.

Based on participant observation and interviews, it is argued that several factors, including exclusion from learning environments, have led to dormancy in the process of knowledge transmission. The establishment of formal primary schools in the refugee camps and many refugees' pursuit of higher education in other countries have also contributed to a reduction in time spent with elders and with others still engaged in camel husbandry, and thus fewer opportunities for knowledge to be passed on. There has also been a noticeable shift in values associated with education, emigration, and exposure to mass media and development schemes; many younger refugees have no interest in camel husbandry, nomadism, and the associated knowledge and cultural heritage and have even come to view these in negative terms, as backward and useless. This is not uncommon within populations who live on the margins economically, socially, and environmentally, and a number of researchers have addressed similar threats to the cultural transmission of knowledge that are associated with abandoned lifestyles in contexts of socio-cultur- 
al change and globalization (Brodt 2001, Maffi \& Woodley 2010, Robinson 2003, Zent 2001).

In spite of these observations, many Sahrawi are increasingly struggling to revitalize their nomadic heritage, practices, and associated knowledge. This process appears to have begun with the Peace Agreement of 1991, the demobilization of Polisario soldiers, the renewed access to cash (e.g., through Spanish civil pensions, remittances, private enterprises, trade), free access to the liberated territories, and strings of rainy years that began in the 1990s. All of these contributed to the resurgence of camel husbandry among nomads and refugees alike and to the revitalization of camel-related knowledge and nomadic heritage (Volpato \& Howard 2014). An increasing number of young refugees are taking up seasonal nomadism and learning the associated traditional knowledge. The competence scores in the CCA of all young refugees that have engaged in seasonal nomadism in recent years were good, suggesting that knowledge transmission is indeed being revitalized amongst such refugees. According to semi-structured interviews conducted with these "new" nomads, they learned about camel forage once they began to engage in camel husbandry and nomadism, acquiring this knowledge from their fathers or uncles if these were once nomads or from peers and co-workers if they were not, employing both vertical and horizontal paths of knowledge transmission. Learning occurs through participation, observation, sharing activities, and instruction, similar to what has been found in other studies (Lozada et al. 2006). Knowledge is once again being used-it can be revitalized in practice because older refugees and former nomads never lost such knowledge, and thus it can now be passed on to a new generation of camel herders.

This revitalization seems to have also included women. In fact, although results confirmed the impression that men have a little more knowledge of camel forage than women, variation is low (about 3\%). This suggests that, in spite of the fact that camel herding is traditionally a male activity, women have almost as much knowledge of camel forage as men. There are two plausible explanations for this: first, historically and contemporarily, men travel for long periods (e.g., to markets) and leave their wives (or other women) in charge of the camel herd, at least part of which remained close to the nomadic camp. Thus, women would know nearly as much as men, at least with regard to the principle forage plants in the customary nomadic territory. Second, women also count among those refugees who acquired camels in the refugee camps (e.g., to provide fresh camel milk to the family or household), and these women would have learned about camel forage and scored better than men who never engaged in camel husbandry.

Processes of revitalization of traditional knowledge have been addressed in the literature, such as those related to bush skills and knowledge among Cree women of Sub- arctic Canada (Ohmagari \& Berkes 1997). In the Sahrawi case, the transmission of traditional camel forage knowledge was partly revitalized because it is crucial to pastoralism and nomadism, which are once again becoming viable livelihood activities as the political and economic conditions change. For many, livestock husbandry represents a means to regain a sense of control over their lives, presenting the possibility of reducing their dependency on food aid. Moreover, the revitalization of livestock husbandry (and the recovery of associated traditional knowledge) reinforces the Polisario's claims to nation-statehood: it provides for national welfare and reduces dependency on food aid via state food production and redistribution, and, drawing upon a shared cultural heritage, it legitimates claims to ethnic unity and national identity (Caratini 2000, Volpato \& Howard 2014).

\section{Conclusions}

This paper has given an account of Sahrawi ethnobotanical knowledge of camel forage plants in Western Sahara with a focus on their identification, properties, and classification. The results show that, emerging from their historical engagement with Western Sahara environment and camel husbandry, the Sahrawi developed a rich and detailed knowledge of camel forage and elaborated strategies of temporal and spatial mobility on the basis of this knowledge in order to provide their camels with the best available forage at any given moment within the accessible pasture areas. Cultural consensus analysis and multidimensional scaling suggest that this knowledge has been retained among nomads and refugees who live or once lived nomadic lives, but its transmission was disrupted among refugees by sedentarization in refugee camps and disengagement with traditional extensive camel husbandry. Thus, the transmission of camel forage knowledge entered into a state that can be characterized as "dormancy," where it was retained by elderly refugees as "theoretical" knowledge and still-nomadic populations as applied knowledge, and thus still available for further transmission to non-knowledge holders given the right conditions. A process of revitalization of knowledge transmission is occurring with refugees' re-engagement with nomadism and pastoralism as political, economic, and even climatic contexts have shifted since the peace accords of 1991 .

Taken together, these results suggest that: (1) knowledge about forage is critical to pastoralists' success and survival in arid environments, since technical husbandry strategies (e.g., movement patterns) depend on this knowledge; (2) in contexts of pastoral population sedentarization, forced displacement, and associated changes in culture and productive activities, transmission of this knowledge may be impaired, leading to its maintenance as theoretical knowledge held by former practitioners, such as elders; and (3) this dormant knowledge may be revitalized and its transmission recovered once it again becomes of material or 


\section{Volpato \& Puri - Dormancy and Revitalization: The fate of ethnobotanical knowledge of camel forage among Sahrawi nomads and refugees}

cultural importance among people who re-engage with past livelihood or environmental management strategies. Of course, in the case of knowledge dormancy, one critical element is the time lag between the loss of relevance and the desire for revitalization; the more time passes, the more likely it is that this dormant knowledge will be permanently lost unless otherwise documented. The time lag might also be important in relation to processes of economic (e.g., competition-driven technological change, change in market demand), cultural (change in cultural values and expectations), and ecological change (e.g., variation in species distribution and communities, in climatic conditions). The longer the time span and the greater the rapidity of change, the more traditional knowledge will need not only to be recovered and transmitted, but also updated or transformed. Sahrawi nomads, who maintained both camels and knowledge of camel forage throughout the war, have also helped refugees to re-initiate camel husbandry and seasonal nomadism by contributing camels (e.g., as camel loans) and knowledge. This has occurred within a more general re-establishment and revitalization of social relations between nomads and refugees based on former tribal affiliation, kin group, bonds of friendship, or commercial relations (e.g., in marketing fresh camel milk in the refugee camps). In this sense, nomads have served as a reservoir of knowledge and engagement with the desert for refugees, i.e., as a minority holding on to a life "as it should be."

These findings enhance our understanding of the critical role of knowledge among pastoral populations and of its value to refugees in recovering and revitalizing their productive and cultural heritage. While many questions remain, including those concerning interventions that can support refugees' aims and agency, from an academic point of view, much more information is needed to understand such processes of knowledge transformation, and thus follow up studies are recommended to document what happens with this knowledge over the next generation as the revitalization process gains strength or wanes.

\section{Acknowledgments}

We are very thankful to many Sahrawi for the patience, kindness, and hospitality that they have shown during fieldwork. We thank the Italian NGO Africa 70-especially Alessandro Broglia, Sara Di Lello, and Davide Rossiand the Veterinary Service of the SADR (Sahrawi Arab Democratic Republic) and its director Saleh Mohamed Lamin for logistical support and cooperation. We are thankful to Sidahmed Fadel and Luali Lehsan for helping with Hassaniya terms, to Prof. Patricia Howard for her editing and comments on the English text, and to Sara RodríguezArgüelles for editing the Spanish abstract. Our gratitude goes also to the Polisario personnel who accompanied Gabriele Volpato in his travels across the liberated territories of Western Sahara. Funds were granted to Gabriele
Volpato by Ceres Research School of Wageningen University, The Netherlands, as part of his PhD research on the ethnobiological knowledge of Sahrawi refugees. The authors thank the editors of ERA and three reviewers for helpful comments and suggestions.

\section{Literature Cited}

Abdelrahim, J. 2013. La crisis asfixia a los saharauis. El País 27 April 2013. Madrid, Spain.

Alexiades, M.N. \& J.W. Sheldon, eds. 1996. Selected Guidelines for Ethnobotanical Research: A field manual. New York Botanical Garden, Bronx, New York, U.S.A.

American Anthropological Association. 1998. Code of Ethics of the American Anthropological Association. Accessed 15 March 2006. www.aaanet.org/profdev/ethics/ upload/ethicscode1998.pdf.

Anderson, E.N., D.M. Pearsall, E.S. Hunn \& N.J. Turner, eds. 2011. Ethnobiology. John Wiley \& Sons, Hoboken, New Jersey, U.S.A.

Barrera, I., M.E. Ron, S. Pajaron \& R. Sidi Mustapha. 2007. Sahara Occidental. Plantas y usos. Usos y costumbres del pueblo saharaui relacionados con los recursos vegetales. Estudio etnobotánico del Sahara Occidental. Universidad Complutense de Madrid y Ministerio de Cultura de la República Árabe Saharaui Democrática, Madrid, Spain.

Benchelah, A.C., H. Bouziane, M. Maka \& C. Ouahes. 2000. Fleurs du Sahara. Voyage ethnobotanique avec les Touaregs du Tassili. Ibis Press-Atlantica, Paris, France.

Berkes, F., J. Colding \& C. Folke. 2000. Rediscovery of traditional ecological knowledge as adaptive management. Ecological Applications 10(5):1251-1262. dx.doi. org/10.1890/1051-0761(2000)010[1251:ROTEKA]2.0. $\underline{\mathrm{CO} ; 2}$

Berlin, B. 1992. Ethnobiological Classification: Principles of categorization of plants and animals in traditional societies. Princeton University Press, Princeton, New Jersey, U.S.A.

Bernard, H.R. 2006. Research Methods in Anthropology: Qualitative and quantitative approaches. $4^{\text {th }}$ edition. AltaMira Press, Lanham, Maryland, U.S.A.

Bhatia, M. 2001. The Western Sahara under Polisario control. Review of African Political Economy 28(88):291301. www.roape.org/088/15.html

Borgatti, S.P. 1996a. ANTHROPAC 4.0. Analytic Technologies, Natick, Massachusetts, U.S.A. 
Borgatti, S.P. 1996b. Methods Manual for ANTHROPAC 4.0. Analytic Technologies, Natick, Massachusetts, U.S.A.

Boyer, J.R. 1962. Contribution à L'étude de L'élevage Camelin au Sahara Occidental: Le Regueibat et son chameau. Thèse doctorat vétérinaire. No. 3. Faculté de Médecine de Paris, Ecole Nationale Veterinaire D'Alfort, Paris, France.

Brodt, S.B. 2001. A systems perspective on the conservation and erosion of indigenous agricultural knowledge in central India. Human Ecology 29(1):99-120. dx.doi. org/10.1023/A:1007147806213

Broglia, A. \& G. Volpato. 2008. Pastoralism and displacement: Strategies and limitations in livestock raising by Sahrawi refugees after thirty years of exile. Journal of Agriculture and Environment for International Development 102(1-2):105-122.

Brooks, N., I. Chiapello, S. Di Lernia, N. Drake, M. Legrand, C. Moulin \& J. Prospero. 2005. The climate-environmentsociety nexus in the Sahara from prehistoric times to the present day. The Journal of North African Studies 10(34):253-292. dx.doi.org/10.1080/13629380500336680

Caratini, S. 1989a. Les Rgaybat (1610-1934). 1: Des chameliers à la conquete d'un territoire. L'Harmattan, Paris, France.

Caratini, S. 1989b. Les Rgaybat (1610-1934). 2: Territoire et société. L'Harmattan, Paris, France.

Caratini, S. 2000. Système de parenté Sahraoui. L'impact de la révolution. L'Homme 154-155:431-456.

Caro Baroja, J. 1955. Estudios Saharianos. Consejo Superior de Investigaciones Cientificas, Madrid, Spain.

Cavalli-Sforza, L.L., M.W. Feldman, K.H. Chen \& S.M. Dornbusch. 1982. Theory and observation in cultural transmission. Science 218(4567):19-27. dx.doi. org/10.1126/science.7123211

Correra, A. 2006. Dynamique de L'utilisation des Ressources Fourrageres par les Dromadaires des Pasteurs Nomades du Parc National du Banc d'Arguin (Mauritanie). Ecologie et gestion de la biodiversité, Museum National d'Histoire Naturelle de Paris, Paris, France.

Cunningham, A.B. 2001. Applied Ethnobotany: People, wild plant use and conservation. People and Plants Conservation Manuals. Earthscan Publications Ltd, Oxfordshire, U.K.

Dedenis, J. 2005. La Mobilité Régionale de la Société Sahraouie des Camps de Réfugiés. Une ressource pour un mieux-vivre? MIGRINTER (Migrations internationales, espaces et sociétés), Université de Poitiers, Poitiers, France.

DNV-RASD. 2007. Census 2007. Dirección Nacional de Veterinaria, Ministerio de Salud Pública Sahrawi, República Árabe Democrática Sahrawi, Rabuni, Polisario refugee camps.

Dobignard, A., F. Jacquemoud \& D. Jordan. 1992a. Matériaux pour la connaissance floristique du Sahara occidental et de l'Anti-Atlas méridional. I. Pteridophyta à Rosaceae. Candollea 47(1):113-179.

Dobignard, A., F. Jacquemoud \& D. Jordan. 1992b. Matériaux pour la connaissance floristique du Sahara occidental et de l'Anti-Atlas méridional. II. Leguminosae à Compositae. Candollea 47(1):397-481.

Doménech, A. 1946. Algo Sobre el Río de Oro. Madrid, Spain.

Ellen, R., S.J. Lycett \& S.E. Johns. 2013. Understanding Cultural Transmission in Anthropology: A critical synthesis. Berghahn Books, Oxford, U.K.

Farah, K.O., D.M. Nyariki, R.K. Ngugi, I.M. Noor \& A.Y. Guliye. 2004. The Somali and the camel: Ecology, management and economics. Anthropologist 6(1):45-55.

Farid, M.F.A. 1989. Water and minerals problems of the dromedary camel (an overview). Options Méditerranéennes - Série Séminaires 2:111-124.

Fernandez-Gimenez, M.E. 2000. The role of Mongolian nomadic pastoralists' ecological knowledge in rangeland management. Ecological Applications 10(5):1318-1326. dx.doi.org/10.1890/1051-0761(2000)010[1318:TROMNP 12.0.CO;2

Folke, C. 2004. Traditional knowledge in social-ecological systems. Ecology and Society 9(3):7. www.ecologyandsociety.org/vol9/iss3/art7/

Gast, M., J.L. Maubois \& J. Adda. 1969. Le Lait et les Produits Laitiers en Ahaggar. Arts et Metiers Graphiques, Paris, France.

Gauthier-Pilters, H. 1961. Observations sur l'ecologie du dromedaire dans le Sahara Nord-Occidental. Mammalia 25(2):195-280. dx.doi.org/10.1515/mamm.1961.25.2.195

Gauthier-Pilters, H. \& A.I. Dagg. 1981. The Camel: Its evolution, ecology, behavior and relationship to man. The University of Chicago Press, Chicago, Illinois, U.S.A.

Guinea, A. 1945. Aspecto Forestal del Desierto: La vegetación leñosa y los pastos del Sahara español. Institu- 


\section{Volpato \& Puri - Dormancy and Revitalization: The fate of ethnobotanical knowledge of camel forage among Sahrawi nomads and refugees}

to Forestal de Investigaciones y Experiencias, Madrid, Spain.

Guinea, A. 1948. Catálogo razonado de las plantas del Sahara español. Anales del Jardín Botánico de Madrid 8:357-442.

Herz, M. 2013. From Camp to City: Refugee camps of the Western Sahara. ETH Studio Basel, Lars Müller Publishers, Zürich, Switzerland.

International Society of Ethnobiology. 2006. ISE Code of Ethics (with 2008 additions). ethnobiology.net/code-of$\underline{\text { ethics/ }}$

Johnson, D.L. 1993. Nomadism and desertification in Africa and the Middle East. GeoJournal 31(1):51-66. dx.doi. org/10.1007/BF00815903

Lebrun, J.P. 1998. Catalogue des plantes vasculaires de la Mauritanie et du Sahara Occidental. Boissiera 55:1322.

Leriche, A. 1953. Phytothérapie maure. De quelques plantes et produits vegetaux utilisées en thérapeutique. Mémoires de l'Institut Français d'Afrique Noire 23:267-306.

Lozada, M., A. Ladio \& M. Weigandt. 2006. Cultural transmission of ethnobotanical knowledge in a rural community of northwestern Patagonia, Argentina. Economic Botany 60(4):374-385. dx.doi.org/10.1663/00130001(2006)60[374:CTOEKI]2.0.CO:2

Maffi, L. \& E. Woodley. 2010. Biocultural Diversity Conservation: A global sourcebook. Earthscan, London, U.K.

Nakao, K. \& A.K. Romney. 1984. A method for testing alternative theories: An example from English kinship. American Anthropologist 86(3):668-673. dx.doi.org/10.1525/ aa.1984.86.3.02a00090

Niamir-Fuller, M. 1998. The resilience of pastoral herding in Sahelian Africa. Pp. 250-284 in Linking Social and Ecological Systems: Management practices and social mechanisms for building resilience. Edited by F. Berkes, C. Folke \& J. Colding. Cambridge University Press, Cambridge, U.K.

Ohmagari, K. \& F. Berkes. 1997. Transmission of indigenous knowledge and bush skills among the Western James Bay Cree women of Subarctic Canada. Human Ecology 25(2):197-222. dx.doi.org/10.1023/A:1021922105740

Ould Mohamed Baba, A.S. 2006. Los fitónimos más frecuentes en el dialecto Hassā-niyyä. Anaquel de Estudios Árabes 17:173-188.
Ozenda, P. 1991. Flore et Végétation du Sahara. 3rd edition. Centre National de la Recherche Scientifique, Paris, France.

Pieroni, A. \& I. Vandebroek. 2007. Traveling Cultures and Plants: The ethnobiology and ethnopharmacy of migrations. Volume 7, Studies in Environmental Anthropology and Ethnobiology. Berghahn Books, Oxford, U.K.

Puri, R.K. 2011. Documenting local environmental knowledge and change. Pp. 126-152 in Conducting Research in Conservation: A social science perspective. Edited by H. Newing. Routledge, London, U.K.

Quinlan, M. 2005. Considerations for collecting freelists in the field: Examples from ethnobotany. Field Methods 17(3):219-234. dx.doi.org/10.1177/1525822X05277460

Robinson, F. 2003. Distribution and Transmission of Traditional Botanical Knowledge in the Changing Social Environment: A study of a Kenyah-Dayak community in interior Borneo. Master's thesis, School of Anthropology and Conservation, University of Kent, Canterbury, U.K.

Romney, A.K., S.C. Weller \& W.H. Batchelder. 1986. Culture as consensus: A theory of culture and informant accuracy. American Anthropologist 88(2):313-338. dx.doi. org/10.1525/aa.1986.88.2.02a00020

Ross, N., D. Medin \& D. Cox. 2007. Epistemological models and culture conflict: Menominee and EuroAmerican hunters in Wisconsin. ETHOS 35(4):478-515. DOI:10.1525/eth.2007.35.4.478

Ryan, G.W., J.M. Nolan \& P.S. Yoder. 2000. Successive free listing: Using multiple free lists to generate explanatory models. Field Methods 12(2):83-107. dx.doi. org/10.1177/1525822X0001200201

San Martin, P. 2010. Western Sahara: The refugee nation. University of Wales Press, Cardiff, Wales.

Scoones, I., M. Melnyk \& J. Pretty. 1992. The Hidden Harvest: Wild foods and agricultural systems. A literature review and annotated bibliography. The Sustainable Agriculture Programme and International Institute for Environment and Development, London, U.K.

Smith, J.J. 1993. Using ANTHROPAC 3.5 and a spreadsheet to compute a free-list salience index. Cultural Anthropology Methods Journal 5(3):1-3.

Thornton, T.F. \& N. Manasfi. 2010. Adaptation-Genuine and spurious: Demystifying adaptation processes in relation to climate change. Environment and Society: Advances in Research 1(1):132-155. dx.doi.org/10.3167l ares.2010.010107 
Turner, N.J. \& K.L. Turner. 2008. "Where our women used to get the food": Cumulative effects and loss of ethnobotanical knowledge and practice; case study from coastal British Columbia. Botany 86(2):103-115. dx.doi. org/10.1139/B07-020

Volpato, G. \& P. Howard. 2014. The material and cultural recovery of camels and camel husbandry among Sahrawi refugees of Western Sahara. Pastoralism: Research, Policy and Practice 4(7).

Volpato, G., P. Kourková \& V. Zelený. 2012. Healing war wounds and perfuming exile: The use of vegetal, animal, and mineral products for perfumes, cosmetics, and skin healing among Sahrawi refugees of Western Sahara. Journal of Ethnobiology and Ethnomedicine 8(49). dx.doi. org/10.1186/1746-4269-8-49

Volpato, G., D. Rossi \& D. Dentoni. 2013. A reward for patience and suffering: Ethnomycology and commodification of desert truffles among Sahrawi refugees and nomads of Western Sahara. Economic Botany 67(2):147-160. dx.doi.org/10.1007/s12231-013-9234-7

Volpato, G. \& A. Waldstein. 2014. Eghindi among Sahrawi refugees of Western Sahara. Medical Anthropology 33:160-177. dx.doi.org/10.1080/01459740.2013.844129
Weller, S.C. 1998. Structured interviewing and questionnaire construction. Pp. 365-409 in Handbook of Methods in Cultural Anthropology. Edited by H.R. Bernard. AltaMira Press, Walnut Creek, California, U.S.A.

Weller, S.C. \& R. Baer. 2002. Measuring within- and between-group agreement: Identifying the proportion of shared and unique beliefs across samples. Field Methods 14(1):6-25. dx.doi.org/10.1177/1525822X02014001002

Wickens, G.E. 1995. Role of Acacia species in the rural economy of dry Africa and the Near East. FAO Conservation Guide 27, Food and Agriculture Organization, Rome, Italy.

Wilson, R.T. 1989. The nutritional requirements of camel. CIHEAM Options Méditerranéennes - Série Séminaires 2:171-179.

Zent, S. 2001. Acculturation and ethnobotanical knowledge loss among the Piaroa of Venezuela: Demonstration of a quantitative method for the empirical study of TEK change. Pp. 190-211 in On Biocultural Diversity: Linking language, knowledge, and the environment. Edited by $L$. Maffi. Smithsonian Institution Press, Washington, D.C., U.S.A. 